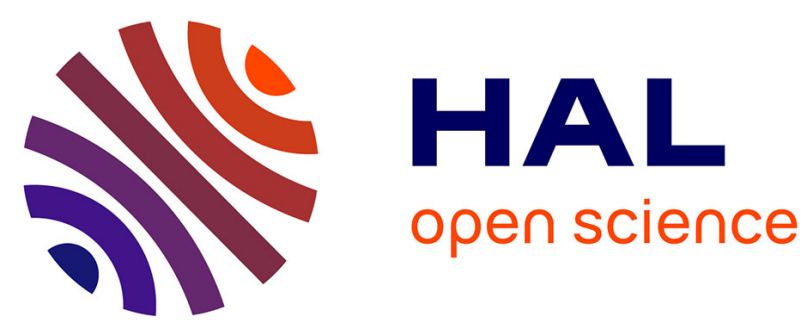

\title{
Numerical Characterization of Landing Gear Aeroacoustics using Advanced Simulation and Analysis Techniques.
}

\author{
S. Redonnet, S. Ben Khelil, J. Bulté, G. Cunha
}

\section{- To cite this version:}

S. Redonnet, S. Ben Khelil, J. Bulté, G. Cunha. Numerical Characterization of Landing Gear Aeroacoustics using Advanced Simulation and Analysis Techniques.. Journal of Sound and Vibration, 2017, 403, p. 214-233. 10.1016/j.jsv.2017.05.012 . hal-01570622

\section{HAL Id: hal-01570622 \\ https://hal.science/hal-01570622}

Submitted on 28 Nov 2019

HAL is a multi-disciplinary open access archive for the deposit and dissemination of scientific research documents, whether they are published or not. The documents may come from teaching and research institutions in France or abroad, or from public or private research centers.
L'archive ouverte pluridisciplinaire HAL, est destinée au dépôt et à la diffusion de documents scientifiques de niveau recherche, publiés ou non, émanant des établissements d'enseignement et de recherche français ou étrangers, des laboratoires publics ou privés. 


\title{
Numerical characterization of landing gear aeroacoustics using advanced simulation and analysis techniques
}

\author{
S. Redonnet*, S. Ben Khelil, J. Bulté, G. Cunha \\ ONERA, The French Aerospace Lab, Châtillon F-92322, France
}

With the objective of aircraft noise mitigation, we here address the numerical characterization of the aeroacoustics by a simplified nose landing gear (NLG), through the use of advanced simulation and signal processing techniques. To this end, the NLG noise physics is first simulated through an advanced hybrid approach, which relies on Computational Fluid Dynamics (CFD) and Computational AeroAcoustics (CAA) calculations. Compared to more traditional hybrid methods (e.g. those relying on the use of an Acoustic Analogy), and although it is used here with some approximations made (e.g. design of the CFD-CAA interface), the present approach does not rely on restrictive assumptions (e.g. equivalent noise source, homogeneous propagation medium), which allows to incorporate more realism into the prediction. In a second step, the outputs coming from such CFD-CAA hybrid calculations are processed through both traditional and advanced post-processing techniques, thus offering to further investigate the NLG's noise source mechanisms. Among other things, this work highlights how advanced computational methodologies are now mature enough to not only simulate realistic problems of airframe noise emission, but also to investigate their underlying physics.

\section{Introduction}

Noise annoyance by aircraft is now officially identified as the major obstacle to sustainable air traffic growth. Therefore, all stakeholders involved in the development of aircraft systems or components are now focusing on practical ways (e.g. technological breakthroughs) to reduce the acoustic signature left by their products. This requires to first mastering the noise phenomena that may occur in realistic situations, which generally implies deploying important research efforts, whether it is from a theoretical, an experimental or a computational point of view. Since acoustics is a complex discipline, part of such efforts is devoted to the continuous development of advanced techniques, which are mandatory for the noise physics can be measured, simulated and/or analyzed still better. In particular, one more and more relies on the numerical simulation, which constitutes a powerful means of investigation, when used to complement experimentation. This, however, requires to constantly develop advanced solving and post-processing techniques, which are needed to simulate and then to investigate the sound generation and/or propagation phenomena occurring in realistic situations.

The noise signature by an aircraft includes two main contributions, respectively of propulsive and non-propulsive origins. The first one, namely the engine noise, is due to all propulsive devices (turbofan or turboprop), whereas the second one,

\footnotetext{
* Corresponding author.

E-mail address: stephane.redonnet@onera.fr (S. Redonnet).
} 


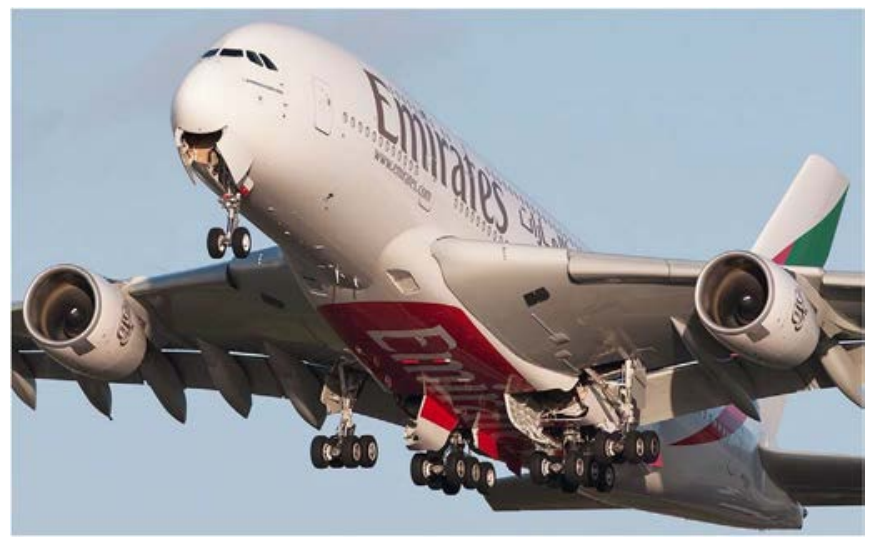

Fig. 1. A380 aircraft with landing gears deployed. Source: Emirates airline and Manchester airport.

namely the airframe noise, is induced by the airframe and its appendages (fuselage, wings, slats, flaps, landing gears, cavities, etc.). Although the engine noise accounts for a dominant portion of the overall aircraft noise during take-off, the airframe noise component becomes equally important during the approach for landing, when the engine thrust is considerably reduced. In particular, a major airframe noise source on large transport aircraft is generated by the landing gears, whose number and size are constantly increased (e.g. A380, see Fig. 1). The underlying mechanisms of noise emissions by landing gears are complex, as shown by various efforts to characterize them via experimentation [1-6] and/or numerical simulation [7-18]. As will be seen hereafter, some of these past experimental and numerical efforts offered a ground for the present work, which constitutes a step further towards the characterization of the aeroacoustics by landing gears.

More precisely, some of these experimental and numerical past efforts dedicated to the characterization of landing gear aeroacoustics were mutualized through the Benchmark for Airframe Noise Computations (BANC), which is an international initiative ${ }^{1}$ that seeks at improving and validating the computational methods dedicated to airframe noise. Among other things, the BANC initiative took direct benefit from the so-called LAGOON project, ${ }^{2}$ whose objective was to acquire an experimental database associated with elementary landing gear configurations, so that computational methods dedicated to landing gear noise predictions can be thoroughly validated. Within this framework, an experimental campaign was thus carried out; focusing on both the aerodynamics and the acoustics of a simplified nose landing gear (NLG), aero-acoustic dual experiments [2,3] were achieved in ONERA's aerodynamic (F2) and anechoic (CEPRA 19) facilities, respectively (see Section 2). The 14th and 15th AIAA-CEAS Aeroacoustics Conference provided an opportunity to thoroughly detail these two experimental campaigns and subsequent results [2,3], which the present third author actively contributed to. In addition, lately, part of such experimental database was disseminated internationally through the BANC workshop (as Category 5), allowing then numerous research teams to validate their numerical capabilities with respect to the prediction of landing gear aeroacoustics [19].

In that regard, several simulations of the NLG configuration were conducted at ONERA, all being performed following a hybrid strategy, ${ }^{3}$ according to which the noise generation and propagation stages are split and solved successively (so as to oppose to the direct approach, where both are computed simultaneously). As a first step of such hybrid scenario, the noise generation stage was simulated through 3D unsteady compressible CFD (Computational Fluid Dynamics) calculations [11], which relied on the so-called ZDES (Zonal Detached Eddy Simulation) approach [20,21], and for which ONERA's elsA solver [22] was used. Such effort, which was achieved by the present second author, was documented at the occasion of the 17th and 18th AIAA-CEAS Aeroacoustics Conferences [12,13], and is detailed in Section 3. As for what had been done regarding the experimental database, and upon the proposal of the present first author, part of such CFD dataset was then disseminated through the BANC workshop (as Category 8), thus allowing various Research Centers (e.g. NASA, JAXA, ONERA) and Universities (e.g. Old Dominion Univ., Campinas Univ.) to benchmark the computational techniques more specifically employed for handling the acoustic propagation stage to be used as a second step of the hybrid scenario [23].

To this end, and contrarily to what was achieved by all other teams (that classically employed a traditional hybrid approach relying on an Integral Method (IM) such as the Acoustic Analogy by Ffowcs-Williams \& Hawkings (FWH) [11-18]),

\footnotetext{
1 Sponsored by AIAA and managed by NASA

2 LAnding Gear nOise database for CAA validatiON project, which was supported by Airbus and conducted by several partners (ONERA, DLR, Southampton University).

${ }^{3}$ Despite the recent development of computational tools and resources, it is still extremely challenging to numerically simulate real-life noise problems following a direct manner, i.e. via a single calculation, because the computational resources required to solve all of the relevant scales would be far too high. Therefore, to make the numerical approach tractable in a practical context, the overall acoustic problem is usually decomposed into a set of subproblems that focus on individual sub-regions of the overall spatial domain. Each sub-problem has a specific range of amplitudes and physical scales that can be addressed using a numerical method that is customized to the dominant physics occurring at this stage. Methods involving a mix of techniques in this manner are classified as hybrid approaches for the acoustic prediction.
} 
use was here made of an advanced hybrid approach originally proposed by the present first author [24], and whose noise propagation stage relies on Computational AeroAcoustics (CAA) techniques. Doing so aimed at ensuring a higher fidelity to the acoustic propagation stage, by i) accounting for the noise emission that had been effectively predicted by the CFD stage (rather than to model it via equivalent sources, as implicitly done by IM/FWH techniques), as well as by ii) possibly considering the realistic jet flow characterizing the experiment (rather than to model it via a simplistic homogeneous medium, as also done by IM/FWH techniques). Following that, the CFD-based noise generation stage previously simulated was interfaced with a CAA-based noise propagation one, which was conducted using ONERA's sAbrinA solver [24-27]. The 19th AIAA Aeroacoustics Conference provided an occasion to early document the outcomes of such CFD-CAA hybrid calculations [28], which were then improved further through an adjustment of both the CAA computational set-up derivation and the CFD data manipulation steps [23]. This work, which was achieved by the present first and fourth authors, is detailed in Section 4.

Finally, based on the CFD-CAA numerical signals acquired over dedicated microphone arrays, the NLG noise sources were further characterized through the use of advanced signal processing techniques [29]; more precisely, once acquired over virtual microphone arrays matching those used in the experiments, the CFD-CAA signals were processed following two distinct array methods of source localization, namely Classical Beam Forming (CBF) and Deconvolution Approach for the Mapping of Acoustic Sources (DAMAS). Such work, which was primarily achieved by the present third and first authors, was documented through the 22th AIAA Aeroacoustics Conference [29], and is recalled in Section 5. Again, in the wake of the latter effort, and upon the proposal of the present first and third authors, lately, a benchmark test case was incorporated within the so-called Array Method workshop, which is an international initiative ${ }^{4}$ that seeks at improving the signal processing techniques commonly employed worldwide for identifying aircraft noise sources.

The present article synthesizes the above summarized works, discussing their methodological and phenomenological outcomes. Indeed, although focusing each on a particular aspect of the overall problem (and deserving each at least one dedicated communication), these various works and associated outcomes all targeted the same common objective, which is the numerical characterization of landing gear noise through advanced computational and analysis techniques - a thing that the present article aims at providing a synthetic overview. To this end, the manuscript is organized as follows; Section 2 briefly recalls the LAGooN experimental campaign, whereas Section 3 describes the near-field unsteady aerodynamics calculation and results, as obtained for a given tested LAGooN configuration. In Section 4, focus is put on the acoustic exploitation of these near-field aerodynamic results through a CFD-CAA calculation relying on the advanced hybrid approach. Then, Section 5 presents how dedicated post-processing of these CFD-CAA outputs (or alternative ones) made it possible to further investigate the NLG noise physics. Finally, Section 6 briefly summarizes the outcomes of all these combined efforts, outlining how the present advanced simulation and exploitation techniques shall be employed soon for addressing others realistic problems coming from the aerospace industry.

\section{Background: experimental characterization of the aeroacoustics by a NLG}

Within the LAGooN framework, combined experimental campaigns were carried out, focusing on both the aerodynamics and the acoustics of a simplified landing gear configuration [2,3]; the model geometry was that of a nose landing gear (NLG) of an Airbus A320 aircraft, with a scale factor of 1:2.5 applied, and with only the main elements (leg, strut, wheels) kept (see Fig. 2(a)). The model was considered as isolated, i.e. free of any airframe components (e.g. fuselage). It was tested for a wide range of flight conditions, whether it is in terms of flow speed (8 Mach numbers, $M$, from 0.1 to 0.28 ) or direction (11 yaw angles, $\beta$, from $-12.5^{\circ}$ to $+12.5^{\circ}$ ). The laminar-to-turbulent flow transition was artificially triggered via strips distributed over the NLG constitutive elements.

The aerodynamic measurements were conducted in ONERA's aerodynamic facility F2, which is a subsonic wind tunnel of closed square test section $(1.8 \mathrm{~m} \times 1.4 \mathrm{~m})$ that is characterized by low levels of turbulence $(<0.05 \%)$ and variation in temperature $\left(< \pm 1{ }^{\circ} \mathrm{C}\right)$. Aerodynamic measurements first focused on the wall pressure field, which was explored through an extensive set of steady and unsteady pressures probes that were located on the NLG surface (wheels, strut, axle). In addition, for two specific configurations (corresponding respectively to typical take-off and approach flight phases $M=0.23$ or $0.18, \beta=0^{\circ}$ ), a complete description of the steady/unsteady velocity field occurring around the NLG model was performed using 2D Particle Image Velocimetry (PIV) as well as 2D/3D Laser Doppler Velocimetry (LDV). Finally, for these two configurations, mixed measurements (hot-wire plus 2D LDV) were performed in the wake of the landing gear, so that additional information (e.g. two-point correlations) is provided.

In a second time, the acoustic measurements were achieved within ONERA's anechoic facility CEPRA 19 (hereafter referred to as C19), which is composed of a subsonic round open jet (whose radius can be adjusted to either $1 \mathrm{~m}$ or $2 \mathrm{~m}$ ) exiting within a large anechoic test chamber (quarter sphere room with a radius of $11 \mathrm{~m}$ ) whose walls are covered with absorbing foam (ensuring a 99\% rate of anechoïcity from $200 \mathrm{~Hz}$ to $80 \mathrm{kHz}$ ). First, it was checked that the associated NLG aerodynamic near-field was similar to that recorded within F2 installation, which was done by the means of specific measurements and dedicated simulations (stationary CFD calculations). Then, acoustic measurements focused on the NLG

\footnotetext{
${ }^{4}$ Sponsored by AIAA and managed by NASA.
} 

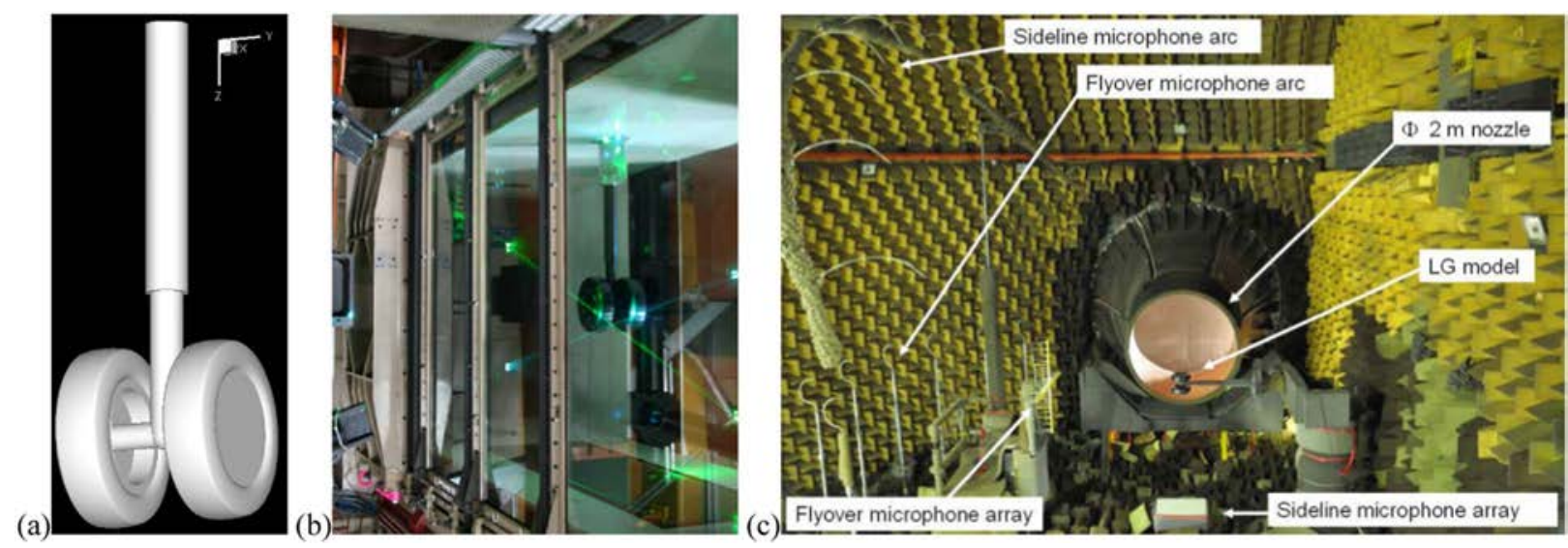

Fig. 2. Experimental characterization of the aeroacoustics by a nose landing gear (NLG) of simplified geometry (a): aerodynamic and acoustic measurement campaigns, as conducted in ONERA's F2 aerodynamic facility (b) and C19 open jet anechoic wind tunnel (c), respectively.

noise radiation, which was recorded through far-field noise directivities. The latter were acquired via $2 \times 12$ microphones that were equally distributed over two arcs located in fly-over and sideline directions, at a distance of approximately 6 m from the mock up (i.e. 20D, with $D$ standing for the NLG wheel's diameter). With the objective of validating computational results, these far-field noise directivities were corrected from various effects by the experimental conditions, such as the refraction by the open jet flow (which was approximated using Amiet's analytical model), the atmospheric absorption by the ambient medium, and the background noise by the facility.

Finally, and regarding more specifically noise source identification purposes, dedicated measurements were performed in both F2 and C19 facilities, so as to record the acoustic signals over microphone arrays of various designs and locations. More precisely, these F2 (resp. C19) phased array measurements relied on a star-shape (resp. cross-shape) array that was composed of 120 (resp. 41) microphones, and that was located 2.6D (resp. 6.5D) away from the gear in both the fly-over and the sideline directions.

\section{Numerical characterization of the NLG noise generation via unsteady CFD}

As said above, following the hybrid philosophy employed here, the NLG noise generation was simulated first, which was achieved through a 3D unsteady compressible CFD calculation.

\subsection{Numerical strategy and computational set-up}

The numerical strategy used for addressing such a typical aerodynamic noise generation step relied on the so-called Zonal Detached Eddy Simulation (ZDES) proposed by Deck [20,21]. This approach constitutes an improved version of the Detached Eddy Simulation (DES) that was originally developed by Spalart et al. [30], and whose basic idea is to combine the features - and thus, to benefit from the respective advantages - of both unsteady Reynolds Averaged Navier-Stokes (URANS) and Large Eddy Simulation (LES) techniques. Indeed, whereas URANS is of low computational cost but is more appropriate for predicting attached flows, LES is able to accurately capture separated flows, but is of higher computational cost. By switching from URANS to LES whenever needed within a same computation, DES offers a mix between both techniques, thus allowing simulating at a reasonable cost most of unsteady aerodynamic problems (e.g. massively separated flows of any Reynolds numbers). In its standard original version, however, DES was too strongly driven by its URANS component, which induced possible bias in the proper ignition of the separated flow dynamics (e.g. delays in the development of vortical structures). To overcome this issue, Deck proposed the ZDES, ${ }^{5}$ which relies on an a priori assignation of the computational areas to be devoted to either URANS or DES [20], so that both techniques are activated wherever needed, thus minimizing the risk of Modelled Stress Depletion (MSD) issues. As a result, in ZDES, URANS mode switches very quickly into LES, thus limiting the delay in the proper development of separated flows (e.g. mixing layers instabilities, vortical structures).

In the present case, since the NLG flow detachments were assumed to mostly occur in the gear median plane ( $x=0)$, and because of methodological constraints, ${ }^{6}$ only the downstream part of the computational box $(x>0)$ was assigned ZDES, its upstream counterpart $(x<0)$ being devoted to URANS (see Fig. 3(b)). Please, note that the laminar/turbulent transition was artificially ignited at the exact same NLG locations where the tripping devices had been set in the experiments. Note also

\footnotetext{
${ }^{5}$ Note that alternative improved versions of DES also exist, such as the so-called Detached DES (DDES) by Spalart et al. [31].

${ }^{6}$ Namely the manual allocation of the RANS to LES switch that ZDES was characterized by, at the time the calculation was achieved (a constraint that was relaxed lately [21]).
} 

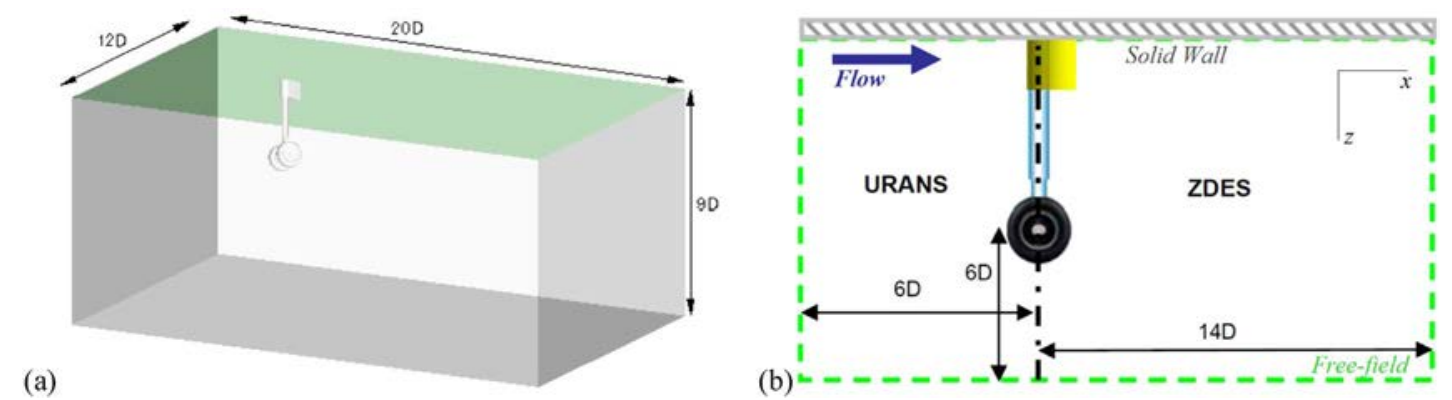

Fig. 3. Numerical characterization of the aeroacoustics by the NLG: near-field noise generation, as simulated via CFD. Sketch of the computational domain (a) and detail of the CFD numerical strategy (b). NLG axle is centered at the origin of the axes.

(a)

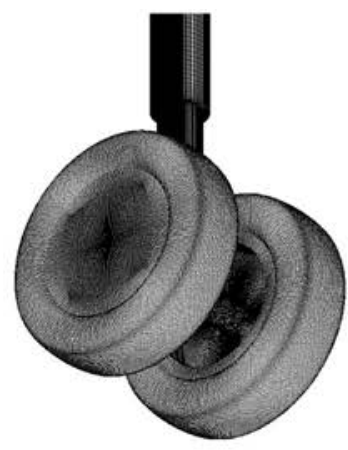

(b)

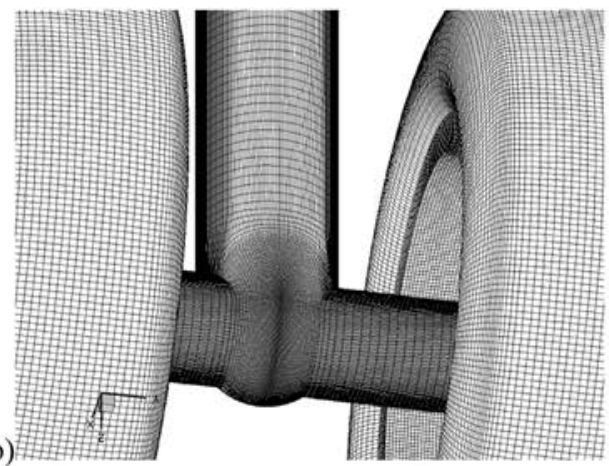

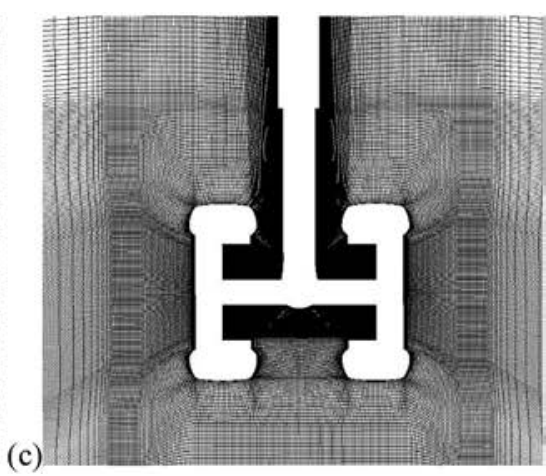

Fig. 4. Numerical characterization of the aeroacoustics by the NLG: near-field noise generation, as simulated via CFD. Details of the structured/multi-blocks CFD grid, (a), (b) and (c).

that the profile element that had been used in the experiments for mounting the NLG leg onto the F2 test section wall was accounted for in the simulation, since it was deemed to possibly impact the flow.

The computational domain was designed so that it extends away from the model up to a distance of $20 D \times 12 D \times 9 D$ in the axial, lateral and vertical directions, respectively (see Fig. 3(a)). With the view of enhancing the similarity of the CFD computational set-up with both F2 experiment (regarding near-field aerodynamics) and C19 test (regarding far-field acoustics), boundary conditions (BC) were set so that only the F2 closed test section wall on which the NLG had been mounted is accounted for in the simulation. In other words, the latter wall was applied a rigid BC (slip, i.e. Euler flow), whereas all the other boundaries of the computational box were allotted an as-like free-field radiating $\mathrm{BC}$ (based on characteristics).

The computation was conducted with the help of the elsA solver [22], which is a structured code that solves the full compressible Navier-Stokes equations using a cell-centered finite-volume approach. In the present case, the latter approach relied on second order modified AUSM+(P) upwind schemes and a second order time integration (here based on $5 \mathrm{~N}$ subiterations per physical time step, and an implicit LU-SSOR scheme). Given the structured nature of elsA solver, the computational domain was meshed with a multi-block conformal grid gathering 111 blocks, for a total of 34 million points (see details in Fig. 4). Such mesh was designed so that the boundary layers are well resolved (with a $y+$ of less than 2) and the early propagation of acoustic waves up to $2.5 \mathrm{kHz}^{7}$ is ensured in the vicinity of the wheels area - a limit beyond which the grid was coarsened, for saving computational resources.

Several computations were conducted so as to simulate the various flight conditions that had been experimentally tested. In the following, however, focus is put on that particular configuration corresponding to a typical approach flight phase $\left(M=0.18, \beta=0^{\circ}\right)$, whose associated Reynolds number (based on $D$ ) was $1.21 \times 10^{6}$; once initialized with a proper flow field solution (coming from a preliminary RANS calculation based on the Spalart-Allmaras turbulence model), the ZDES simulation was conducted for a total physical duration of $150 \mathrm{~ms}$, with a time step of $0.5 \mu \mathrm{s}$. After a $50 \mathrm{~ms}$ transient phase was washed out from the computational domain, a physical time of about $100 \mathrm{~ms}$ was acquired for exploitation. Corresponding to a convection of the flow over a distance of $20.6 \mathrm{D}$, this $100 \mathrm{~ms}$ simulation time required about $130,000 \mathrm{CPU}$ hours of parallel calculation via 256 processors of a scalar SG.

\footnotetext{
${ }^{7}$ With a nominal cell size of $\Delta_{\mathrm{xyz}} \sim 3 \times 10^{-3} \mathrm{~m}$, i.e. ensuring 40 points per apparent wavelength $(f=2.5 \mathrm{kHz}, M=0.18)$.
} 
(a)

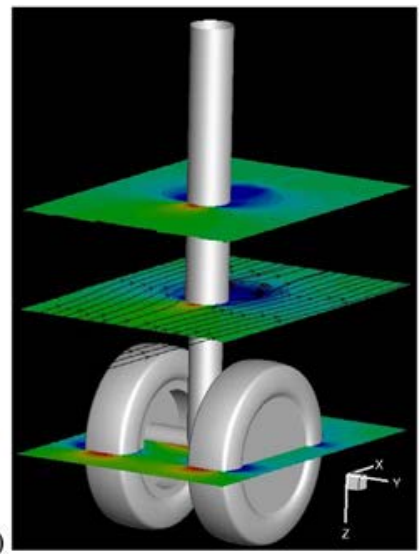

(b)

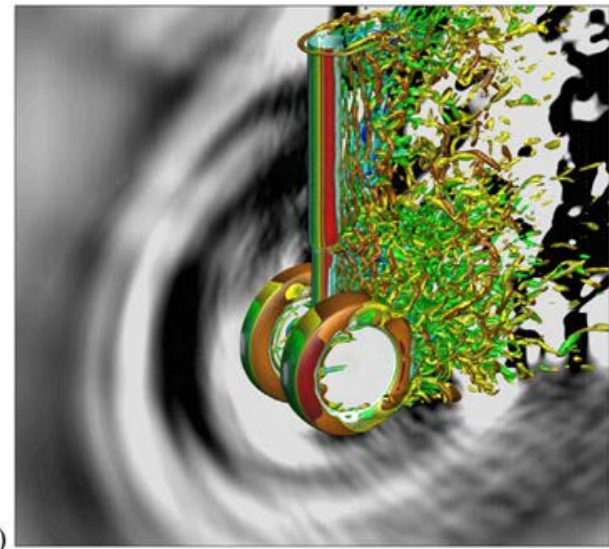

Fig. 5. Numerical characterization of the aeroacoustics by the NLG: near-field noise generation, as simulated via CFD. Steady mean flow (a) plotted as pressure and velocity streamlines, and unsteady perturbations (b) depicted in terms of Q-criterion iso-surfaces and instantaneous perturbed pressure. (For interpretation of the references to color in this figure, the reader is referred to the web version of this article).

(a)

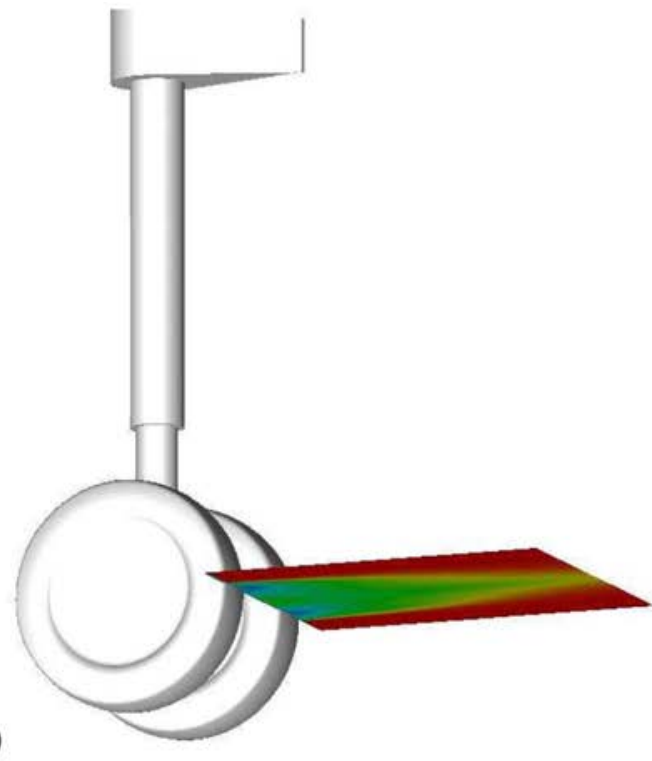

(b)

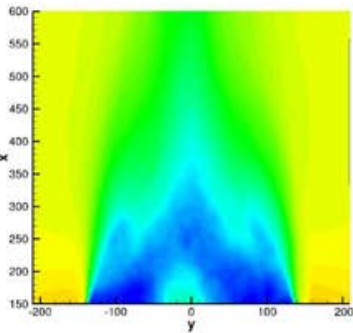

(d) (c)

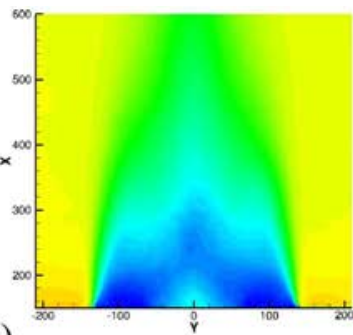

(e)

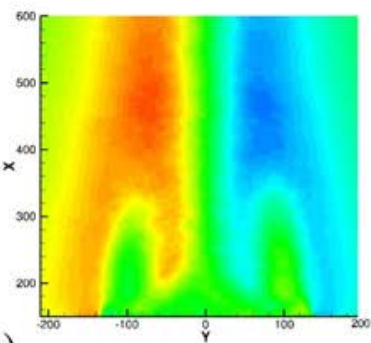

Fig. 6. Numerical characterization of the aeroacoustics by the NLG: near-field noise generation, as simulated via CFD. Validation of the calculation results via direct comparison against experimental outputs: Particle Image Velocimetry (PIV) maps within a $z=0$ median plane located downstream the axle (a), for both the streamwise and the transverse mean velocity field, as computed and measured; (b) streamwise/computed, (c) streamwise/measured, (d) transverse/computed, (e) transverse/measured.

\subsection{Computational results and analysis}

As an illustration, Figs. 5-7 depict some of the ZDES results obtained; first, Fig. 5(a) displays the aerodynamic steady flow-field occurring around the NLG, here plotted in terms of stream wise velocity. The unsteady counterpart of the previous steady flow-field is provided on Fig. 5(b), depicted here in terms of Q-criterion iso-surfaces (colored by the streamwise velocity) as well as of instantaneous pressure fluctuations. There, one can clearly distinguish the fully three dimensional nature of the resulting unsteady flow, noticing in particular how the vortical structures generated downstream the gear are numerically ignited without any visible delay - which illustrates the ability of ZDES to efficiently reproduce the expected dynamics of flow separation. Apart from that, one can also distinguish the early propagation of those acoustic waves that radiate in all directions, once emitted by the gear.

The above computational results were favorably validated through extensive comparisons against F2 measurements, both regarding the steady and the unsteady flow fields. Among other things, comparisons focused on the distribution of both the time-averaged/static and the unsteady/fluctuating pressure around the NLG elements. Comparisons also focused on the velocity field occurring immediately downstream the gear, whose steady (profiles, maps) and unsteady (spectra, spacetime correlations) components were favorably compared against experimental ones. 


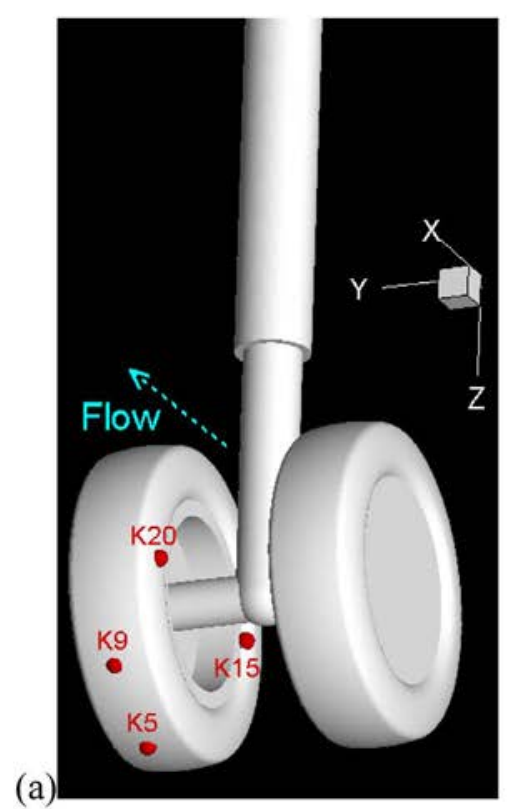

(b)

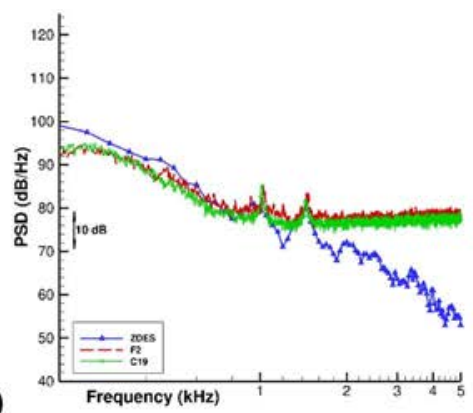

(d)

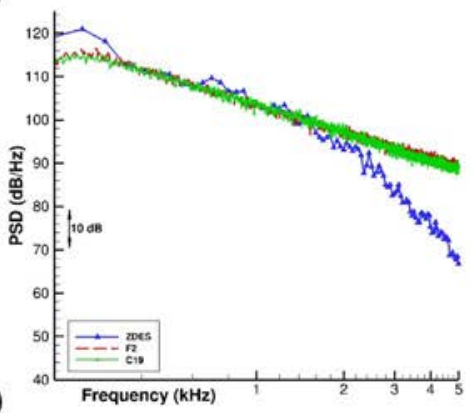

(c)

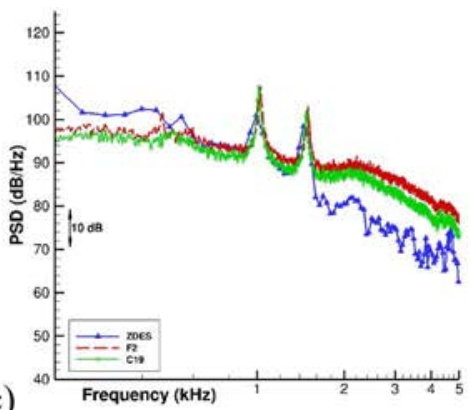

(e)

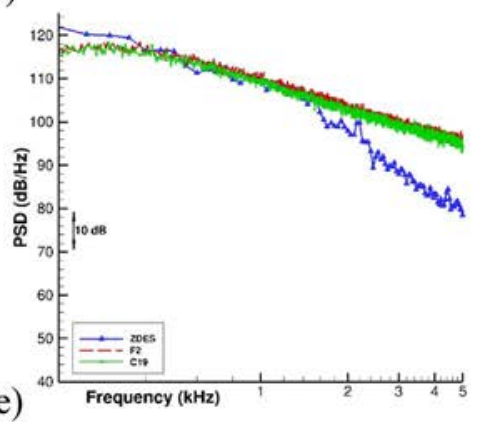

Fig. 7. Numerical characterization of the aeroacoustics by the NLG: near-field noise generation, as simulated via CFD. Validation of the calculation results via direct comparison of the Power Spectral Density (PSD) computed and measured for four probes of the right wheel (a): probes K5 (b), K15 (c), K9 (d) and K20 (e). Instantaneous perturbed pressure computed (in blue lines and symbols) as well as measured in F2 (in red dashes) and C19 anechoic (in green lines and symbols) facilities. (For interpretation of the references to color in this figure legend, the reader is referred to the web version of this article).

As an illustration, Fig. 6 depicts the mean velocity maps that were computed (center side) and PIV-measured (right side) within a $z=0$ plane located immediately downstream the gear (left side). As one can see, the computation reproduces fairly well the experiments, with flow patterns that are very similar to those measured, whether it is in terms of stream wise or transverse velocities. This result is rather satisfactory, if one considers how complex this particular flow region is because of the way the wake emitted by the axle interacts with the downstream parts of the wheels (half cavity and tires, etc.).

Another example of these validation efforts is provided on Fig. 7, which compares the spectra (Power Spectral Density, PSD) associated with the CFD unsteady pressure field recorded at various locations of the NLG's right wheel surface to those coming from either F2 or C19 experiments. First of all, one can notice how similar the outputs from both experiments are for all four locations, which confirms a posteriori the effective reproducibility of the aerodynamic and acoustic tests. Then, one can see how the numerical results match the experimental ones, whether it is in terms of trends (decaying slope) or absolute values (amplitude) - at least, over that frequency range which was well resolved by the numerical method. Indeed, as expected, beyond its cut-off frequency limit $(2.5 \mathrm{kHz})$, the calculation departs from the experiments, which may obviously be attributed to the filtering effects induced by the CFD grid. For some probes, one can also notice that the simulation slightly over-predicts the measured levels over the lower part of the frequency range. This discrepancy might actually rather come from the way the CFD results were acquired/post-processed rather than from the way they were computed; indeed, for practical reasons, the CFD time series had to be stored with a 1:10 time sampling applied, which may have logically led some of the higher frequencies to be back aliased onto the lower part of the spectrum [32]. This, not to mention the highly disproportionate frequency bandwidths respectively used for deriving (via Fourier-Transforms) the experimental and numerical spectra, given the disparate duration of both signals.

Most remarkable result of this comparative exercise is the fact that, for some probes, both the computation and the experiments exhibit two similar high intensity tonal features (of approximate frequency $1 \mathrm{kHz}$ and $1.5 \mathrm{kHz}$ ). By analyzing the entire wall pressure dataset, as well as by corroborating the resulting observations with those deduced from the noise directivities that had been measured in the far-field, it appeared that these tonal emissions radiated more in the sideline directions, and were to be related to resonances coming from the wheels' inner cavities. This last conclusion was inferred from the fact that i) both tonal emissions emerged more for those probes that were located in the inner part of the wheels (e.g. probe K15 on Fig. 7(a)), ii) their respective frequency did not depend on the speed of the incoming flow (see Fig. 8 (a) and Fig. 8(b)), but iii) was rather linked to the NLG wheels dimensions through a simple law $f_{k}=k \cdot \mathrm{c}_{\mathrm{o}} / 2 L^{8}-$ with $f_{k 1}$ and $f_{k 2}$ corresponding here to the first and second frequency $\left(\sim 1 \mathrm{kHz}\right.$ and $1.5 \mathrm{kHz}$ ), when considering for the associated lengths $L_{1}$ and $L_{2}$ either the wheel's cavity diameter or the tires' inter-space distance (see Fig. 8(c), in red and blue respectively).

Finally, on this stage, one can notice that the good agreement between computational and experimental results

\footnotetext{
${ }^{8} k$ and $c_{o}$ standing here for the wavenumber and the sound velocity, respectively.
} 

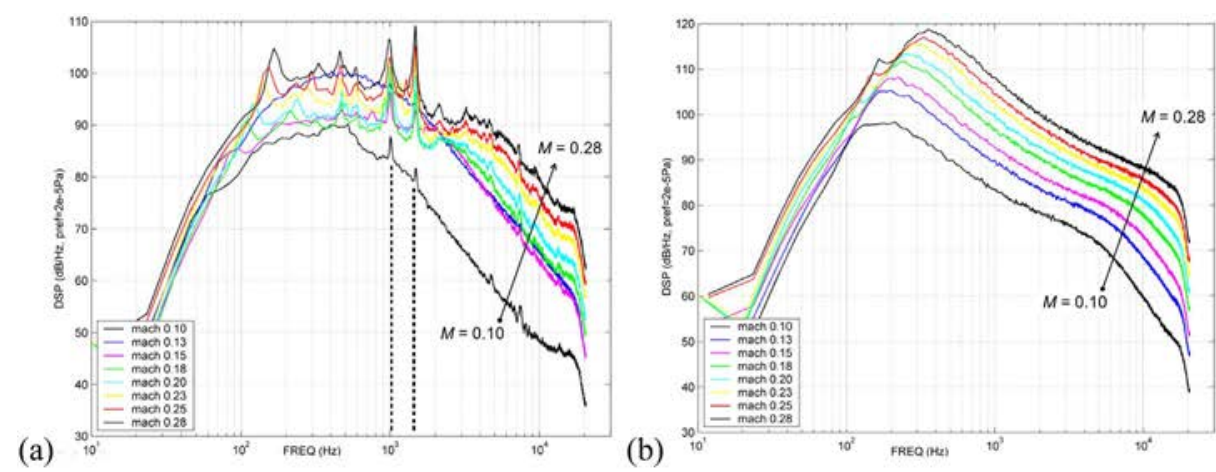

(c)

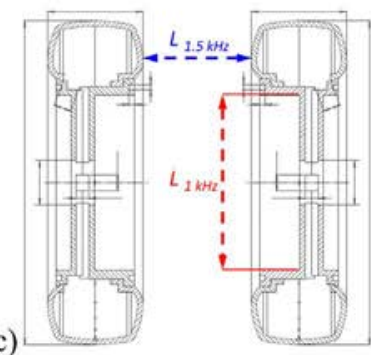

Fig. 8. Experimental characterization of the aeroacoustics by the NLG: further investigation of the two tonal emissions $(f \sim 1 \mathrm{kHz}$ and $1.5 \mathrm{kHz})$, highlighted here by the vertical dashes in (a). PSD of the unsteady pressure recorded on probe K15 (a) and K9 (b), with dependence towards the flow speed ( $M=0.10-$ 0.28 , by increments of 0.03 ). (c) Characteristic lengths of the wheels' cavities (diameter and spanwise extent), for a dimensional analysis and physical interpretation of the tonal emissions in terms of resonances coming from the gear's wheels inner cavity. (For interpretation of the references to color in this figure legend, the reader is referred to the web version of this article).

legitimates a posteriori the somehow hybrid computational set-up (partly open test section) that had been defined so as to match as closely as possible both F2 and C19 configurations at the same time, and whose bias does not seem to have impacted the flow solution.

\section{Numerical characterization of the NLG noise radiation via CFD-CAA}

Still accordingly to the hybrid philosophy used here, after the NLG noise had been numerically generated via CFD, it was further propagated through CAA.

\subsection{Numerical strategy and computational set-up}

The numerical strategy employed for doing so was that of the advanced hybrid method which was originally proposed and extensively advocated by the present first author, and whose idea is to interface unsteady CFD and CAA (Computational AeroAcoustics) [24]. Indeed, over the last years, ONERA devoted significant research means to the development of a robust and accurate hybrid method whose propagation stage would be based on a CAA step, rather than on an Integral Method (e.g. Acoustic Analogy) one - as usually done. The motivation behind this was to enhance both the accuracy and the versatility of the noise propagation stage, thanks to the two main advantages offered by a CAA method compared to IM ones, i.e. its ability to both i) handle the actual noise signal to be CFD-simulated over the near-field sound generation area(s) and ii) account for the installation effects that may alter such noise signal during its near- to far-field propagation. To this end, several research actions were achieved, which all served the same purpose whilst focusing each on a particular aspect of the problem; early works $[25,26]$ focused on the CAA stage itself, leading to the development of ONERA's CAA solver sAbrinA [24-27]. Then, specific research actions [33] consisted in developing the so-called Non Reflective Interface (NRI) technique, which allows the CAA-based propagation stage to be forced ${ }^{9}$ with any given acoustic signal whilst offering to handle acoustic backscattering phenomena (for the overall hybrid method is compatible with many situations of interest). Finally, follow-on research actions [34] consisted in optimizing the latter hybrid method for it can cope with all stringent constraints that are dictated by real-life applications (e.g. CPU requirements) without being jeopardized by some of their unavoidable side effects (such as the signal degradation to which unsteady CFD data are subjected when manipulated for being acoustically exploited [32]). This last effort led to several methodological innovations, such as the so-called Specific Preservation Criteria (SPC) [34], the Interpolation by Parts (IBP) technique [35], and the Intrinsically Optimized Finite Differences (IOFD) schemes [36]. Thanks to the various outcomes they led to, all these studies made it possible to derive an advanced CAA-based hybrid approach that integrates and connects altogether the diverse aspects of the aeroacoustic problem whilst accounting for their interactions. Such approach, which was recently documented in a dedicated journal article [24], was validated through an application to various problems of increasing complexity [24,34], being then applied to the present NLG configuration.

Following that, the CFD-based noise generation stage described in Section 3 was interfaced with a CAA-based noise propagation one, the latter being conducted using sAbrinA solver. One can here remind that sAbrinA solver [24-27] is a structured, time-accurate CAA code that solves either the full or the linear Euler equations in a conservative and perturbed form (with a splitting of the complete variables into a 'frozen' mean flow and a 'fluctuating' perturbation). The solver

\footnotetext{
${ }^{9}$ In a so-called 'one-way' (or unilateral) CFD $\rightarrow$ CAA fashion, given that acoustic waves are assumed to be too weak to retro-act onto the unsteady aerodynamic flow they are generated by (which actually justifies the small perturbation (sole) hypothesis underlying CAA methods [25,26]).
} 

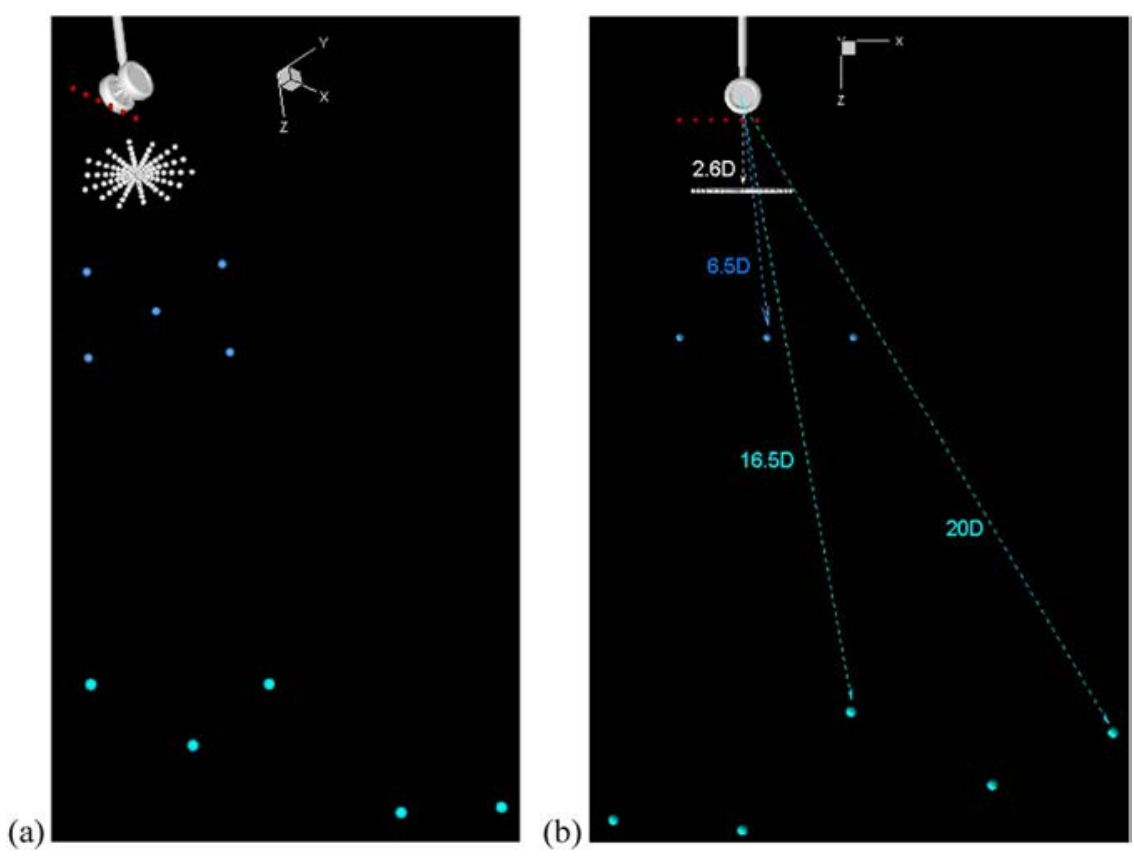

Fig. 9. Numerical characterization of the aeroacoustics by the NLG: near- to far-field noise propagation, as simulated via CFD-CAA. Images (a) and (b); two views of the virtual microphones used in the CAA stage, for the validation and exploitation of the CFD-CAA unsteady results.

employs finite-difference (FD) operators involving high-order spatial derivatives and filters, as well as a 3rd-order, multistage, Runge-Kutta time-marching scheme. The code handles multi-block structured grids with one-to-one interfaces, and is fully parallelized using the Message Passing Interface (MPI) standard. Finally, the solver includes the usual boundary conditions (reflection by solid walls, non-reflecting/free-field radiation, etc.), as well some unique to specific applications - such as the above mentioned NRI technique, which was used in the present effort for forcing the CAA stage with the CFDoriginated NLG noise signal. Aside from that, the present effort also took direct benefit from the above mentioned IOFD derivation and filtering schemes, whose higher accuracy (with respect to their standard counterpart of same stencil width and/or leading order) made it possible to drastically reduce the computational cost (see below). Finally, used was also made of the SPC criterion, which allowed optimizing the computational set-up for a better preservation of the CFD noise signal (see below).

The CAA computational set-up was derived as follows; first, the CAA domain was designed so that it extends up to the regions of interest (e.g. areas where noise experimental measurements were available), whereas remaining small enough for the computation and restitution (e.g. visualization) efforts are kept reasonable enough, both in CPU time and memory. Since reaching all of the experimental microphones was deemed to be impractical, such CAA domain was restricted to a cubic box of dimensions $[-7.5 D, 12.5 D] \times[-12.5 D, 12.5 D] \times[-2.5 D, 21.5 D]$, which encompassed most of the flyover microphones (that were located in either the near-, mid- or far-field, at a distance of approx. 2.6D, 6.5D and 20D from the NLG axle, respectively - see Fig. 9).

This computational domain was meshed with a Cartesian grid, i.e. a grid offering both better propagative features (accuracy, stability, etc.) and an easier handling (topology, etc.) than a curvilinear mesh. This was made possible thanks to the initial choice of not incorporating the NLG geometry within the CAA stage, which would have considerably increased the meshing/computational efforts without bringing any substantial extra information (considering the relatively small dimensions of the gear). Such CAA grid was derived analytically, being designed in such a way that it guarantees a correct propagation of acoustic waves over the frequency range of interest $(0-3 \mathrm{kHz})$, whereas allowing a possible capture of the mean flow effects (e.g. convection, refraction); considering the 15-point stencil IOFD schemes to be used for the CAA consumption, a minimum of 4 Points Per Apparent Wavelength (PPW) associated with the maximal frequency $(3 \mathrm{kHz})$ was ensured all over the domain. The CAA grid density was however progressively refined over all regions where a higher spatial resolution was required, such as the areas where the CFD-CAA interface was located (see below). At the end, this heterogeneous Cartesian CAA mesh comprised a total of about 66 million points. One can here notice that if such grid had been designed for a 7-point standard FD scheme (rather than for the 15-point IOFD one used here), it would have led to a prohibitive number of grid cells (more than 1.5 billion of points).

The CFD-CAA interface was located around the sole wheel's area (see Fig. 10), the reasons for doing so being that i) preliminary analyses had revealed that the NLG noise radiation was to be mainly dominated by the contribution coming from the wheels (especially in the flyover direction, which was primarily targeted here), ii) some of the noise waves were likely to have been numerically dissipated along the leg's region (over which the CFD mesh had been coarsened for saving 

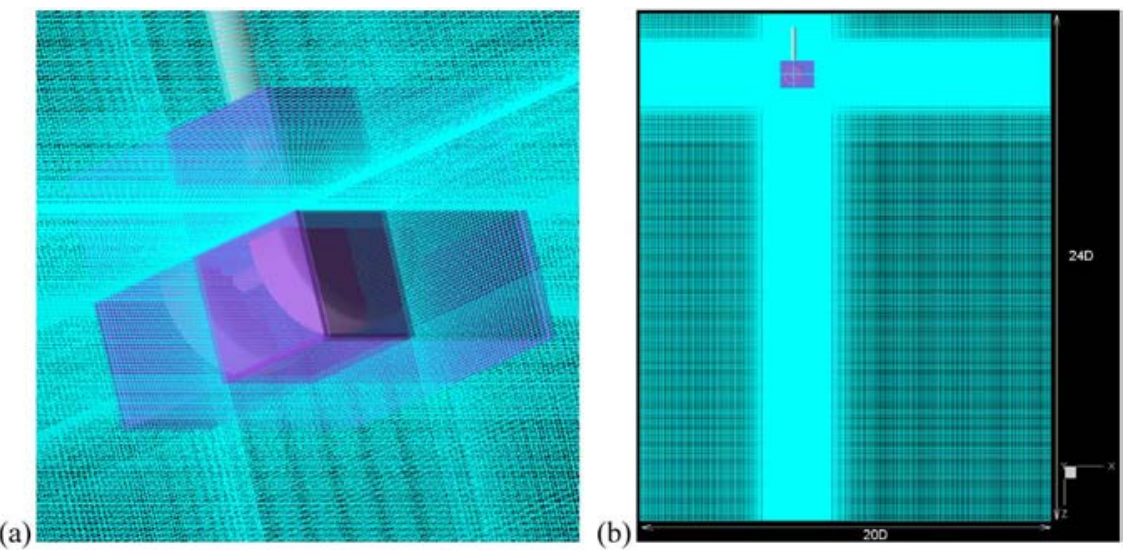

Fig. 10. Numerical characterization of the aeroacoustics by the NLG: near- to far-field noise propagation, as simulated via CFD-CAA. (a) and (b); two views of the Cartesian heterogeneous CAA mesh, along with the CFD-CAA interface (cubic box around the NLG's wheels) that was used for NRI-forcing the CAA stage with the CFD-generated noise signal.

computational resources), and iii) a storage of the CFD unsteady data over both the wheels and the leg regions would have been too prohibitive in terms of memory requirements. The CFD-CAA interface was positioned close enough from the wheels, without possibly getting too much into regions of strong hydrodynamic activity - all this resulting from a compromise to be found between the accuracy of the unsteady aerodynamics stage and the stability of the acoustic one ${ }^{10}$ $[24,37]$. With the view of preserving at best the CFD stored signal from being degraded by the necessary CFD $\rightarrow$ CAA interpolation in space, the CFD-CAA interface was initially set such that its grid point density matches that of the CFD mesh. It was thus designed as a Cartesian envelope constituted of about 800,000 points equally distributed over 6 faces of 5 layers thickness. In order to avoid a prohibitive storage volume, the CFD data were stored with a 1:10 sampling applied in time, such storage being achieved during the second half of the CFD calculation. This led to a total of 14,400 temporal occurrences (corresponding to a physical duration of $72 \mathrm{~ms}$ ) and an overall volume of $0.75 \mathrm{~TB}$. Finally, since it would have been prohibitive to keep all of its constitutive points for the CAA consumption, and based on insights coming from specific analyses (e.g. SCP criterion), the CFD-CAA interface was lightened before it was CAA-exploited, each one of its 6 faces being sampled along its lateral directions (rate of 1:2) whereas being kept as is in its normal direction. In addition, the back and top faces of the CFD-CAA interface were ignored in the CAA calculation, whose grid density would have been far too insufficient for handling accurately enough all of the hydrodynamic occurrences (vortices) such faces were crossed by, because of the wake generated downstream the NLG's leg and axle. ${ }^{11}$ Please, note that the way the previous computational set-up was derived has been more extensively documented in Ref. [28], so as to serve as methodological guidelines regarding CFD-CAA matters.

The CAA consumption was run for a total of 12,000 time steps (i.e. approx. $42 \%$ of the temporal extent of the CFD calculation), which corresponded to a physical duration of $60 \mathrm{~ms}$. The computation was processed in parallel over 480 cores, requiring a CPU time of $45 \mathrm{~h}$. With respect to the total number of grid points (66 millions) and iterations $(12,000)$ involved, this represents an average CPU time of about $98 \mu \mathrm{s} /$ cell/iteration (a non-negligible part of which was devoted to the I/O operations needed for reading the 101 GB of binary data that constituted the CFD-originated source signal to be NRI-forced within the CAA calculation). After the transient had evacuated the CAA computational box (which required approx. 4000 iterations, that is, one third of the calculation), the time history of all perturbed quantities was stored in various points of the computational box, which corresponded to either microphone locations where experimental data had been recorded or to numerical probes were CFD data had been acquired for analysis purposes. Those time signatures were then post-processed, whether it is via classical Fourier analysis (e.g. far-field noise radiation through isolated microphones, see Section 4.2), or via more advanced techniques (e.g. noise source localization through microphone arrays, see Section 5.3).

\subsection{Computational results and analysis}

A first CFD-CAA hybrid calculation focused on the isolated NLG, i.e. it incorporated a uniform mean flow corresponding to a homogeneous and unbounded medium. The purpose here was to validate the CFD-CAA results and underlying methodology through direct comparison against the C19 experimental records (which had been corrected from the refraction

\footnotetext{
${ }^{10}$ For the source signal is preserved at best from inaccuracies occurring either within the noise generation stage (e.g. signal degradation due to excessive dissipation induced by insufficiently accurate CFD schemes) or within the acoustic propagation stage (e.g. signal degradation due a corruption induced by an insufficiently dense CAA grid) - see [24,37] for more detail on this point.

11 One can here specify that, as discussed in [24,37], such an arbitrary removal of part of the CFD dataset may be partially conclusive only, depending on the problem addressed; for some configurations, it may thus be required to NRI-force the CAA stage with the entire CFD source signal. In which case, it would only be needed to densify the CAA mesh for it is able to capture all of the CFD-generated source occurrences (to begin with unsteady aerodynamics ones, e.g. turbulence). This in turn may imply a drastic increase of the CPU price to be paid for the CAA consumption.
} 


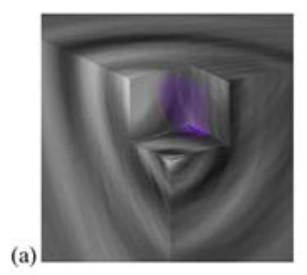

(b)

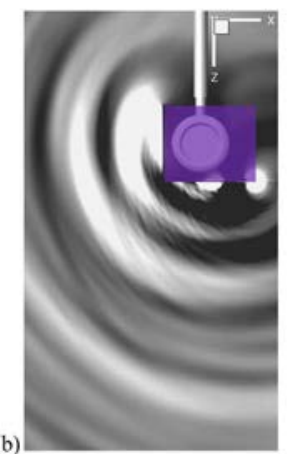

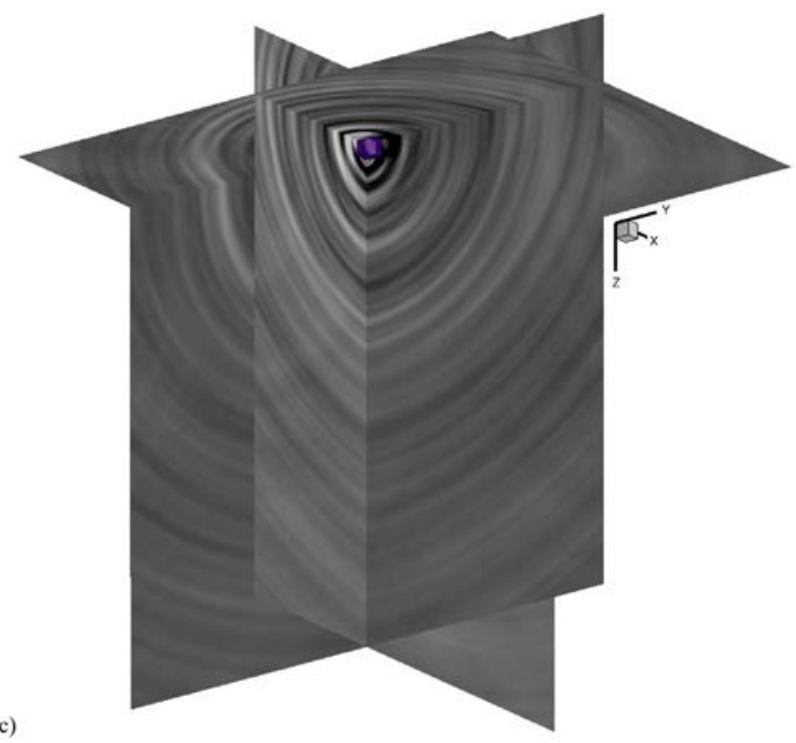

Fig. 11. Numerical characterization of the aeroacoustics by the NLG: near- to far-field noise propagation, as simulated via CFD-CAA. Computation results (instantaneous perturbed pressure), as obtained after the CFD-generated noise signal has been NRI-forced (through the cubic interface, (a) and (b)) and then CAA-propagated up to the far-field (c). Scale: 60 iso-contours from -5 to +5 Pa.

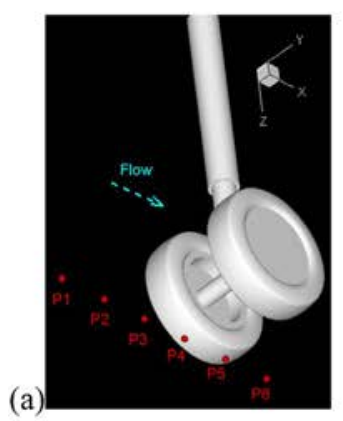

(b)

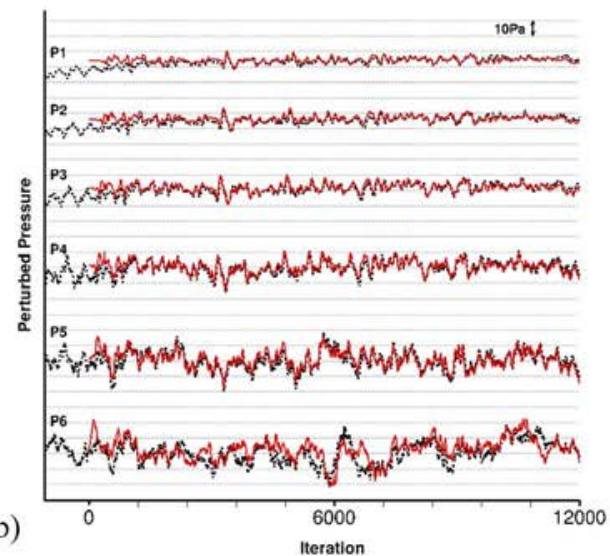

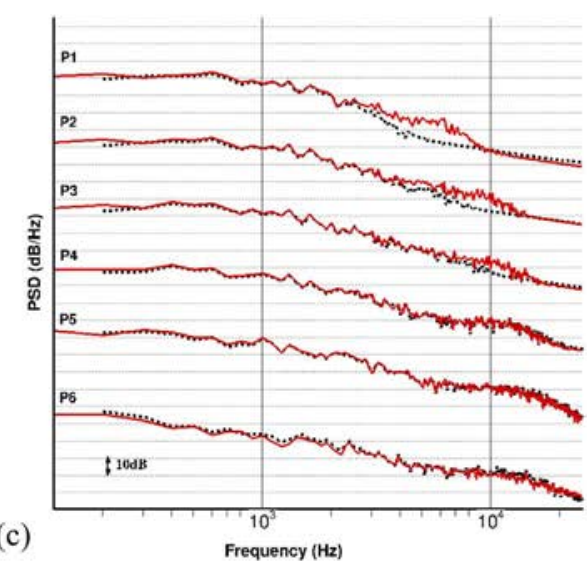

(c)

Fig. 12. Numerical characterization of the aeroacoustics by the NLG: near- to far-field noise propagation, as simulated via CFD-CAA. Early validation of the calculation results via direct comparison of the near-field outputs acquired via either CFD (black dashes) and CFD-CAA (red lines) simulations: time signature (b) and PSD (c) of the instantaneous perturbed pressure recorded for six probes (P1 to P6) located in the vicinity of the right wheel (a). (For interpretation of the references to color in this figure legend, the reader is referred to the web version of this article).

effects by the open jet shear layers), as well as against the numerical outputs coming from more traditional CFD-IM hybrid calculations by NASA, JAXA and ONERA (which could not incorporate any effect by the jet flow) [23]. Fig. 11 displays the instantaneous perturbed pressure field that was obtained at one half of the calculation. As one can see, once forced within the CAA domain via the NRI interface (small cubic box, drawn here in purple), the CFD source signal was CAA-propagated up to the far-field. Here, it is worth noticing that the resulting acoustic emission was somehow irregular, translating the intermittent character exhibited by the CFD dynamics itself.

With the view of validating such CFD-CAA hybrid calculation, several comparison exercises were conducted. First, the efficiency of the CFD-CAA interfacing procedure was checked by verifying that the CFD source signal was properly NRIforced within the CAA domain; on Fig. 11(a), one can appreciate how the patterns of the prescribed source signal (which is plotted here over the CFD-CAA interface) are well recovered within the CAA domain.

This qualitative agreement is confirmed by the quantitative match delivered on Fig. 12, which compares directly the present CFD-CAA outputs (in red) with those that had been initially gathered during the CFD stage (in black), this being done for six identical probes located in the immediate vicinity of the CFD-CAA interface. As one can see on Fig. 12(b), each pair of time signatures exhibit very similar patterns, especially for the probes located upstream (P1 to P4) or right under (P5) the 

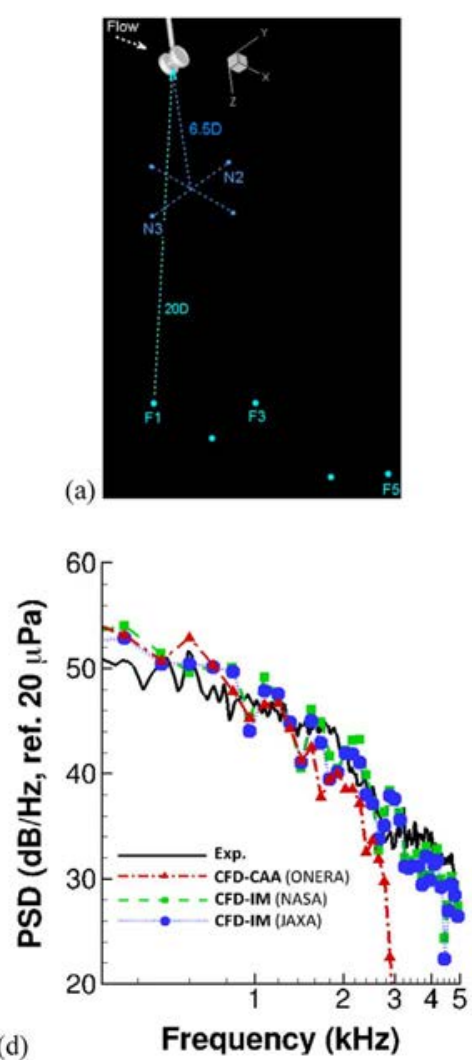

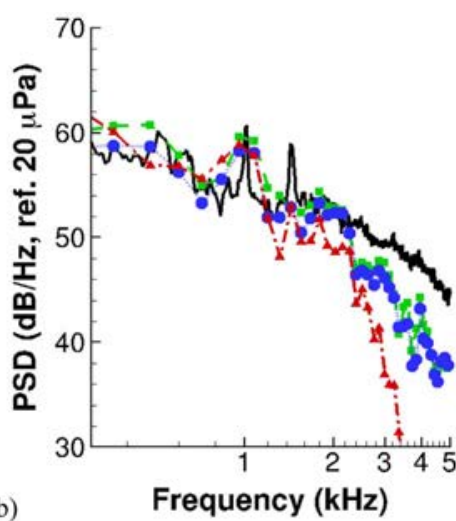

(b)

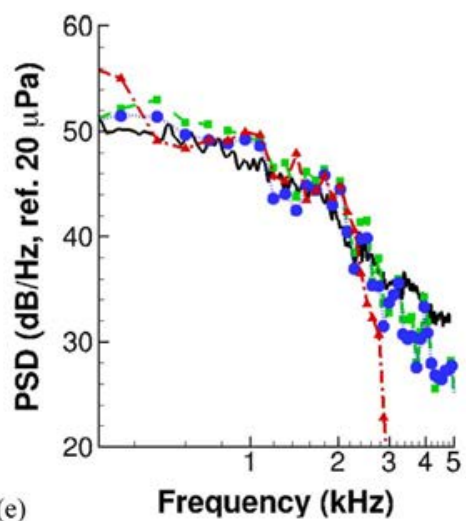

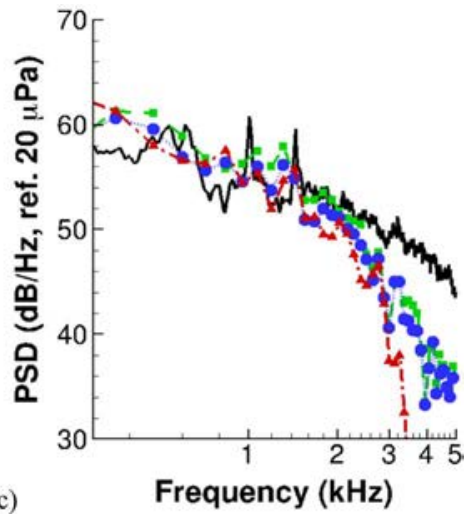

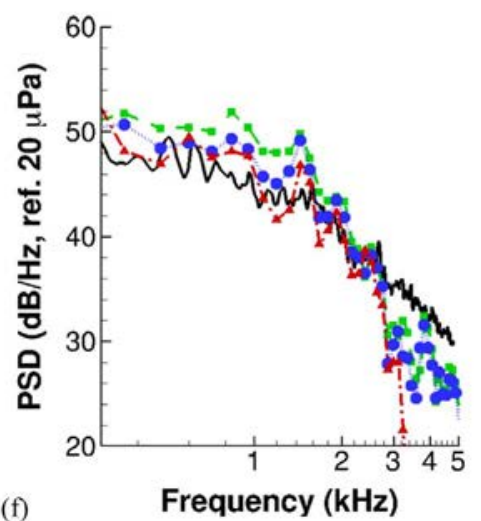

Fig. 13. Numerical characterization of the aeroacoustics by the NLG: near- to far-field noise propagation, as simulated via CFD-CAA. Further validation of the calculation results via direct comparison of the mid- and far-field outputs, as measured through C19 experiment (in black) and simulated via either traditional CFD-IM methods (in blue and green) or the present CFD-CAA approach (in red, with associated $f_{\text {cut-off }}=3 \mathrm{kHz}$ ). PSD of the instantaneous perturbed pressure recorded for two mid-field and three far-field probes microphones (a); probes N2 (b), N3 (c) and F1 (d), F3 (e), F5 (f). (For interpretation of the references to color in this figure legend, the reader is referred to the web version of this article).

NLG axle. For the probe located immediately downstream the gear (P6), the agreement between the two time signatures is less satisfactory because of spurious effects coming from the sporadic impingement by strong unsteady aerodynamic occurrences onto the downstream part of the CFD-CAA interface - a thing that was expected to occur, given the proximity of the latter interface and the gear. Fig. 12(c) depicts the Power Spectral Density (PSD) counterpart of the previous time signature pairs, which was obtained via windowed Fourier transforms (with a frequency accuracy of around $100 \mathrm{~Hz}$ ); as one can see, all pairs of spectra collapse, to the exception of probe P6 (for the reasons evocated above), as well as probes P1 to P3, for which the higher frequency range $(f>3 \mathrm{kHz})$ appear to be less and less resolved by the CFD, as the distance between the probe and the gear increases. This is due to the higher dissipative character of the CFD stage, whose 2nd order finite volume schemes were not accurate enough for propagating the noise signal very far away from the NLG - this, contrarily to the CAA stage, whose IOFD schemes logically ensured a much accurate propagation of the acoustic waves.

Further validation of the CFD-CAA results are provided on Fig. 13, which depicts (in black) the noise spectra radiated in the mid- and far-field (i.e. approximately 6.5D and $20 \mathrm{D}$ away from the mock-up in the flyover direction, see Fig. 13(a)), comparing them favourably to those recorded experimentally (in red). Please, note that for indicative purpose, the same figure also provides (in blue and green) the spectra coming from more traditional CFD-IM hybrid calculations (by NASA and JAXA, respectively [23]). The latter were derived from the same CFD dataset under identical quiescent medium conditions, and using the so-called 'porous surface' FWH integration technique. First of all, one can there appreciate how both CFD-CAA and CFD-IM outputs match experimental results quite closely, at least up to the accuracy limit of the CFD signal (2.5 kHz). Beyond that limit, both CFD-CAA and CFD-IM levels logically decay, the former decreasing however faster than the latter because of the intrinsic cut-off frequency by the CAA grid $(3 \mathrm{kHz})$. Below this limit, and except for some amplitude mismatches occurring here and there (see explanations below), the agreement between all results is fairly good, as both hybrid approaches reproduced correctly the trends of experimental records whether it is in terms of broadband amplitude levels and decay slopes, or tonal peaks.

This is rather satisfactory if one considers how inaccurate the spectra analysis of both CFD-CAA and CFD-IM results was, 
compared to that of the experimental records. ${ }^{12}$ More precisely, whereas the latter had been Fourier Transform (FT) processed with many averaging blocks and a frequency bin width of approx. $10 \mathrm{~Hz}$, the former where FT-processed with only a few averages and a frequency resolution of $120 \mathrm{~Hz}$. In particular, this insufficient accuracy of numerical FTs explains why, for the mid-field lateral microphones (see probes N2 and N3 on Fig. 13(b) and Fig. 13(c), respectively), both CFD-CAA and CFDIM spectra do not exhibit both tonal peaks $(1 \mathrm{kHz}, 1.5 \mathrm{kHz})$ as clearly as their experimental counterpart. Indeed, it was checked that depending on the FT parametrization (e.g. frequency bin width and origin), one or the other of these tonal peak emerged more clearly - but not both at the same time.

One can finally notice how, for the far-field microphone F5 (see Fig. 13(f)), both CFD-IM calculations over-predicted the expected levels, contrarily to the CFD-CAA simulation, which roughly matches the experiment. This certainly comes from the wake-induced spurious effects that were previously evocated; indeed, as explained in Ref. [37], IM propagation methods are as sensitive as CAA ones to these spurious effects, even if the latter occur to a lesser extent, and for different reasons. In the present case, both IM-based calculations relied on the entire CFD dataset, contrarily to the CAA-based counterpart (for which top and back sides of the CFD-CAA interface had been dropped down, see Section 4.1 above). As a consequence, more spurious noises were likely to be IM-propagated up to that particular microphone F5, which was more exposed to them than any other probe, being located further downstream the gear. Oppositely, the last observation justifies a posteriori the fact of having neglected part of the CFD dataset for the CAA consumption, which would have been more importantly impacted by these spurious effects, otherwise.

From a more global perspective, one can underline that the overall variability between all these CFD-CAA and CFD-IM outputs may be attributed to the respective ways the CFD dataset was CAA- or IM-exploited, with intrinsic differences whose nature was both fundamental (e.g. equivalent vs. exact acoustic source, linear vs. non-linear propagation kernel) and practical (e.g. truncation and sampling of the CFD-CAA interface) [37]. However, again, one can appreciate how, despite of all these methodological differences, both CFD-CAA and CFD-IM outputs agree well altogether, and compare favourably with the experiments. This further validates both the CFD-based generation stage and its exploitation via a hybrid approach, whether the latter is traditional (e.g. IM) or more innovative, as the present CAA-based one. As a corollary, this also confirms that the rather strong approximations made for lightening the CAA stage consumption (e.g. sampling and truncation of the CFD-CAA interface) were compatible with the present configuration. This might not be the case for more complex problems whose specificities might impose that the CFD-CAA interface is designed more accurately and/or is made compatible with a crossing by incoming turbulence. Addressing the latter point will be the subject of future work.

\section{Further investigations about the NLG noise physics}

In the wake of the previous CFD-CAA simulation, further investigations were achieved through alternative calculations and/or specific post-processing of the subsequent simulation results.

\subsection{Further investigation about the NLG tonal emissions}

First, the CFD-CAA results previously obtained were post-processed using Fourier Transforms (FT), so that the harmonic field associated with each one of the two tonal emissions $(f=1 \mathrm{kHz}$ and $1.5 \mathrm{kHz})$ is extracted from the resulting overall broadband solution (see Fig. 14). Both tones were found to be characterized by very specific patterns, with acoustic waves ${ }^{13}$ radiating quite differently in both the fly-over and the sideline directions (planes $x z$ and $x y$, respectively). Regarding more particularly the noise emission occurring within the sideline plane (see Fig. 14(b) and Fig. 14(d)), the radiation features appeared as being composed of an asymmetric pattern (which, here, primarily emerges for the first mode, $f=1.0 \mathrm{kHz}$, Fig. 14 (b)), to be combined with a (anti-)symmetric one (which, here, is more visible for the second mode, $f=1.5 \mathrm{kHz}$, Fig. $14(\mathrm{~d})$ ). This corroborates former observations by Casalino et al. [16], who ran the exact same NLG configuration (under slightly different flight conditions, $M=0.23 / \beta=0^{\circ}$ ) via a CFD-IM calculation (whose CFD staged relied on a Lattice-Boltzmann Method, LBM); indeed, following a dedicated analysis of the near-field results they obtained within the wheels area, authors concluded that both $f=1.0 \mathrm{kHz}$ and $f=1.5 \mathrm{kHz}$ tones were characterized by a so-called bistable asymmetric regime, which was primarily constituted by i) an asymmetric mode for which flow structures radiated waves of different energy levels and of opposite phase from both sides of the wheels, whereas alternating their left/right sides behavior - such shift in the left/right cycle going along with ii) a (anti-)symmetric mode of lower intensity and shorter duration. Additional analyses revealed that such complex dynamics resulted from the combination of two distinct noise generation mechanisms, that is, a Rossiter

\footnotetext{
12 Indeed, with respect to the length of the experimental time-series $(20 \mathrm{~s})$, the CFD-generated signal was of much shorter duration (0.06 s). In addition, when further propagated through CAA or IM, and because of the transient time needed for the first wave front to reach the probes location, the effective length of the useful signal that was numerically recorded was even shorter, with respectively $0.05 \mathrm{~s}$ and $0.04 \mathrm{~s}$ for the mid- and far-field microphones, respectively. As a consequence, the spectra analysis of the CAA- and IM-based outputs were much less accurate than the one applied to the experimental data.

13 Whose radiative wavelength was checked to be coherent with the expected frequency: $\lambda \sim 0.34$ and 0.23 , respectively.
} 

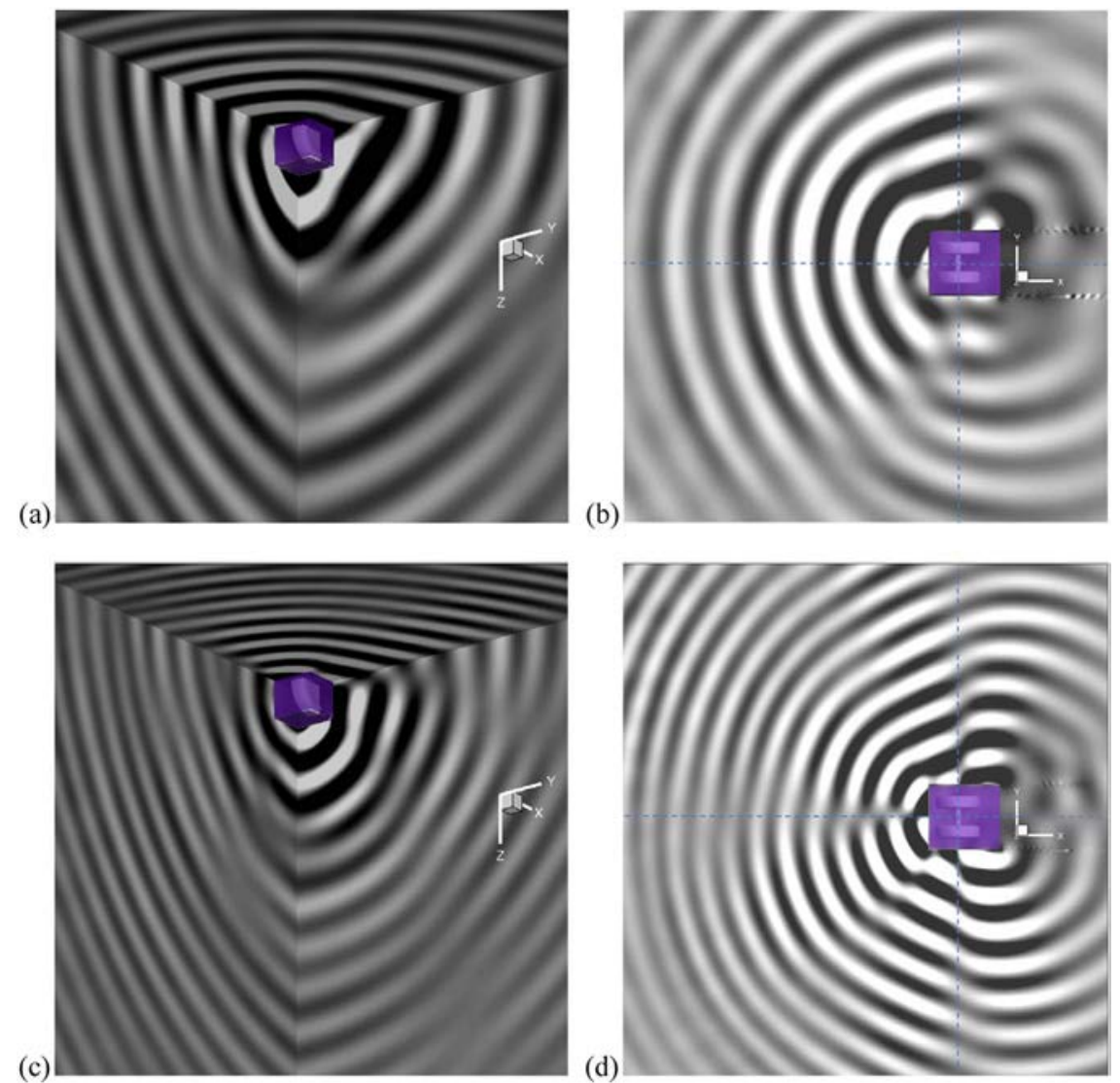

Fig. 14. Numerical characterization of the aeroacoustics by the NLG: near- to far-field noise propagation, as simulated via CFD-CAA. Further investigation about the two tonal noise emissions, via a dedicated post-processing of the calculation results. Harmonic field radiated by the first $(f \sim 1.0 \mathrm{kHz})$ and second $(f \sim 1.5 \mathrm{kHz})$ tones, as obtained by FT-processing the broadband result coming from the CFD-CAA calculation (homogeneous medium). Left side; 3D views of the instantaneous perturbed pressure field associated with the first (a) and second (c) tones. Right side: corresponding radiation patterns within the sideline $x y$ plane for the first (b) and second (d) tones, with the emergence of both an asymmetrical and an anti-symmetrical pattern ( $x z$ and $y z$ axes appear in dashes). Scale: 60 iso-contours from -0.3 to $+0.3 \mathrm{~Pa}$.

feedback loop ${ }^{14}$ by the round cavities (emitting in the flow direction, i.e. $x$ ) and a transverse acoustic resonance by the two facing annular cavities (radiating in the sideline directions, i.e. $y$ ) - the phase interference between both mechanisms making the asymmetrical or (anti)symmetrical features emerge more or less. These features are made clearly visible by the present CFD-CAA outputs, which highlight better the ability of the advanced hybrid method employed here to not only capture the NLG noise dynamics, but to also reveal its underlying mechanisms.

\subsection{Further investigation about the acoustic installation effects by the C19 facility jet flow}

With the view of leaning towards more realism whilst taking full benefit from the present CAA-based hybrid approach, an alternative CFD-CAA hybrid calculation was then ran, for which the NLG was considered as installed in C19 anechoic wind tunnel, i.e. it was sunk within an incoming flow corresponding to that of the anechoic test section. Indeed, contrarily to what was assumed in the previous CFD-CAA calculation (as well as in any other CFD-IM simulation performed so far [7-18]), the flow surrounding the NLG during the acoustic experiment was far from being homogeneous. More precisely, when exiting the nozzle it is initially confined in, C19 jet logically spreaded out itself within the ambient medium, thus leading to the creation of shear layers whose strength goes decreasing with the axial distance. The latter shear layers were likely to have modified the NLG noise radiation, whether it is by deflecting the emitted acoustic waves from their initial propagation paths or by trapping part of their energy within the jet core region $[38,39]$.

Therefore, in order to assess the possible installation effects such particular jet flow may have had onto the experimental noise measurements, the acoustic propagation stage was here enhanced with a background mean flow matching the realistic steady jet occurring in the C19 facility - a thing that only a CAA method could allow doing, since IM techniques can

\footnotetext{
${ }^{14}$ Rather than a Karman vortex shedding by the cylindrical struts (axle or leg).
} 

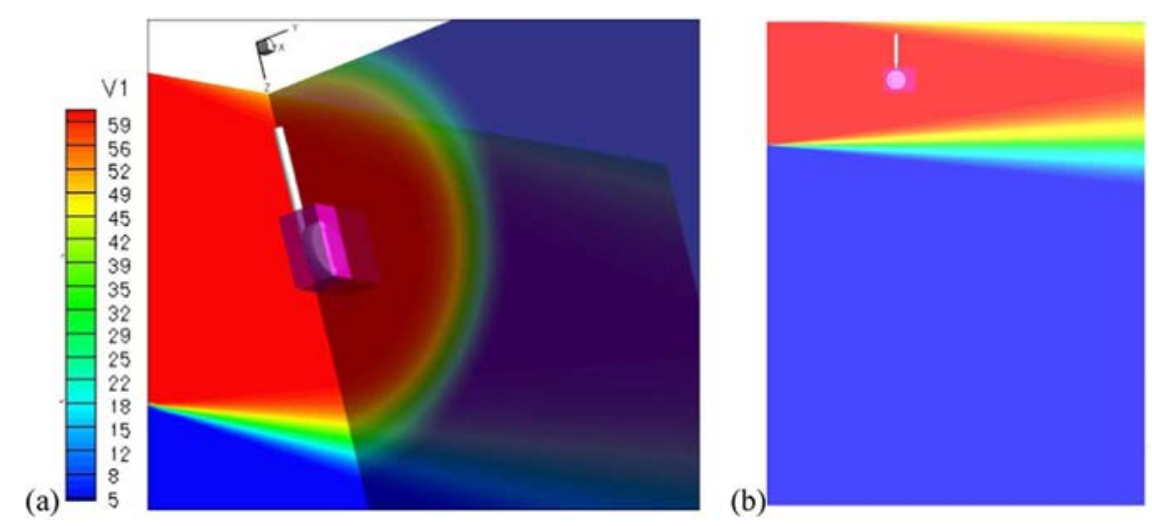

Fig. 15. Numerical characterization of the aeroacoustics by the NLG: near- to far-field noise propagation, as simulated via CFD-CAA. Further investigation about the acoustic installation effects by the C19 anechoic facility jet onto the NLG noise radiation, via an alternative CFD-CAA calculation incorporating a C19-like heterogeneous medium. CAA background mean flow reproducing the spreading out round jet occurring within C19 facility, depicted here in terms of axial velocity (scale: 18 iso-contours from 0 to $60 \mathrm{~m} \mathrm{~s}^{-1}$ ). 3D closer view (a) and 2D cut (b) of the CAA domain.

(a)

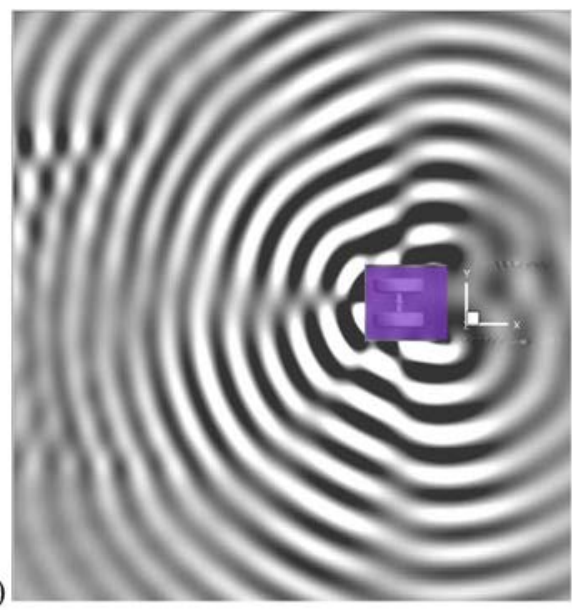

(b)

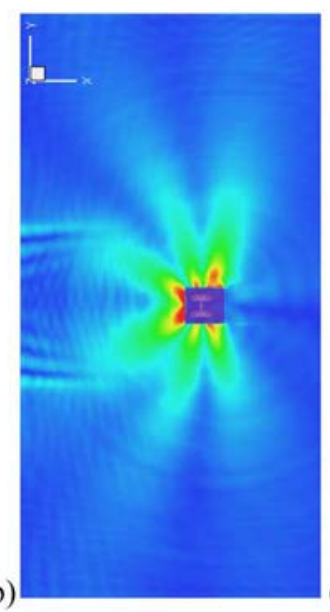

(c)

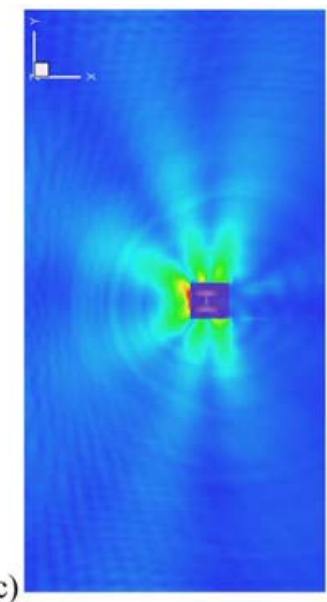

Fig. 16. Numerical characterization of the aeroacoustics by the NLG: near- to far-field noise propagation, as simulated via CFD-CAA. Further investigation of the refraction effects by the anechoic facility jet onto the NLG noise radiation, via an alternative CFD-CAA calculation incorporating a C19-like heterogeneous medium: harmonic field radiated within the sideline $x y$ plane by the second $(f \sim 1.5 \mathrm{kHz})$ tonal emission, as obtained by FT-processing the broadband results coming from the alternative CFD-CAA calculation (heterogeneous medium). Image (a): instantaneous perturbed pressure field (closer view, scale: 60 iso-contours from -0.3 to $+0.3 \mathrm{~Pa}$ ). Images (b) and (c), respectively: Root Mean Square (RMS) counterpart of such perturbed pressure field, compared with that coming from the previous (homogeneous medium) CFD-CAA calculation.

only handle homogeneous media. To this end, and following what had been achieved in a previous effort [33], a C19-like background mean flow was derived analytically, this being done with the help of similarity functions. The latter were designed for spatially evolving with a spreading out angle of $5^{\circ}$, thus corresponding to the growth of the turbulent boundary layer thickness characterizing C19 jet in its ' $2 \mathrm{~m}$ nozzle diameter' configuration (see Fig. 15). Except this particular background mean flow, the CAA computational setup was kept strictly identical to that of the previous CFD-CAA calculation, being in particular NRI-forced with the same CFD-originated source signal, thus allowing to numerically isolating the sole acoustic installation effects by the facility jet.

From a quantitative point of view, the impact induced by such acoustic installation effects onto the far-field noise radiation recorded in the fly-over directions were found to be rather modest, modifying the PSD levels in a very minimal way (less than a few $\mathrm{dB}$ at maximum, depending on the microphone location - cf. probes F1 to F5 on Fig. 13). This, which is coherent with Amiet's theory [39], was expected to happen in such a situation for which i) both the Mach number and the frequency range were rather low, and ii) the microphones were mostly located at the zenith of the noise source (i.e. $90^{\circ}$ with respect to the jet axis). However, more noticeable effects by this C19 jet flow onto the NLG emission were observed to occur in other areas of the propagation domain. As an illustration, Fig. 16, depicts the harmonic perturbed pressure field associated with the second tonal emission $(f=1.5 \mathrm{kHz})$, as delivered by the present alternative CFD-CAA computation.

By comparing this result with that of the baseline (homogeneous medium) previous CFD-CAA computation (see Fig. 14 (d)), one can clearly notice how the noise radiation patterns were now noticeably modified by the jet, especially nearby the 
nozzle exit, where the shear layers were denser. In particular, the interference patterns one can distinguish over this area translate the backscatter effects by the jet shear layers, whose interface deflected towards the jet axis those acoustic waves that reached it with an angle of incidence smaller than a limit value [38,39]. This resulted in a confinement of the acoustic waves, whose energy remained partly trapped within the jet core region. Such effect appears more clearly on the center and right sides of the same Fig. 16, which respectively depict the Root Mean Square (RMS) counterpart of the harmonic perturbed fields associated with both the alternative and the baseline CFD-CAA computations. As one can see, contrarily to when the flow was uniform, the jet trapped a fraction of the NLG noise, leading to a slight increase (resp. decrease) of the acoustic energy levels within (resp. out of) the jet core region.

One can here point out that these observations are fully in line with what can be theoretically predicted [38] and was numerically verified through a dedicated alternative study by the present first and third authors [40], which consisted in numerically assessing (via both Computational AeroAcoustics and Geometrical Acoustics) the acoustic installation effects by various canonical open jet flows onto elementary sources (monopoles of low to high frequencies). Indeed, generally speaking, the effects by open jet flows are primarily driven by the aspect ratio between the noise source wavelength $(\lambda)$ and either i) the shear layer thickness $(\delta)$ or ii) the radius $(r)$ of the jet. Following Ref. [40], such aspect ratio can be expressed through two non-dimensionalized parameters, $\gamma=\delta / \lambda$ and $\beta=r \mid \lambda$, which respectively refer to the refraction effects (that tend to deflect the emitted noise waves away from their initial propagation path, thus modifying the acoustic signature out of the jet) and the confinement effects (that tend to backscatter the emitted noise waves at the shear layer's interface, thus trapping the acoustic energy within the jet core region). Those primary mechanisms and related parameters also depend on other factors such as the apparent wavelength with which acoustic waves propagate along their paths, the relative angle of incidence with which they then hit the shear layer's inner interface, etc. All this may result in complex interaction figures, especially when the configuration is not trivial (as is the case here), because of the jet flow structure (jet's core Mach number and direction, jet's curvature and spreading angle, etc.) and/or the noise emission dynamics (broadband signal, intermittent character, etc.). In such a case, it may be difficult to theoretically predict the overall effects by the open jet flow, which are however likely to be captured via the present CAA-based approach [40]. Here again, it is worth noticing that such a thing could not be achieved by a standard IM-based hybrid approach, because of the underlying hypothesis (e.g. homogeneous propagation medium) its IM stage relies on. All this emphasizes the importance of employing advanced techniques such as the present CAA-based hybrid method for enhancing the fidelity of acoustic predictions, e.g. for numerically assessing the relevance of the analytical corrections [39] that are commonly applied to acoustic data measured in open jet anechoic wind tunnels.

\subsection{Further investigation about the NLG noise sources}

Once their acoustic emission had been numerically simulated via the previous CFD-CAA hybrid computations, the NLG noise sources were further characterized through the use of array analysis techniques, namely Classical Beam Forming (CBF) and Deconvolution Approach for the Mapping of Acoustic Sources (DAMAS [41,42]). The primary objective here was to assess how far noise source localization methods based on array techniques could be transposed from an experimental to a computational context. Indeed, these methods were originally developed - and are now widely used - for experimental purposes, whether it is regarding facility testing, fly-over or in-flight experiments. On another hand, these methods strongly rely on advanced signal processing techniques, whose performances may greatly vary depending on the configuration to be considered (e.g. array geometry employed, frequency range targeted, acoustic signal recorded), because of the possibly illposed nature of the associated inverse problem. In particular, there are legitimate concerns about the variability of a given noise source localization method upon the nature of the acoustic signal it is fed with, depending on if such signal is of long duration but contaminated with extra noises (as generally happens with experiments) or, oppositely, is highly accurate but of very short duration (as usually happens with computations). In addition, among the various existing noise source localization methods, some of them (e.g. CBF) are less accurate but more robust, whereas others (e.g. DAMAS) are of higher accuracy but come along with a greater sensitivity to various factors such as the way of $i$ ) translating the recorded noise signals into cross-spectral densities (e.g. parametric or non-parametric methods) and/or of ii) array-processing them effectively (e.g. focalization area, algorithm convergence, regularization parameters).

With the view of assessing their effective applicability and possible sensitivity within a computational context, both CBF and DAMAS techniques were thus applied to the two previous CFD-CAA hybrid computations, through a post-processing of the CFD-CAA signals that had been stored on purpose over specific virtual microphone arrays matching those used in the experiments.

In order to spare them from being space interpolated (and, thus, possibly degraded), the CFD-CAA signals were stored over locations that slightly approximated those of the experiment (typically, by the closest grid point of the CAA calculation mesh). It was however checked that the location errors to be possibly induced by such an approximation were negligible, given the high density of the CAA grid over the mid-field areas where the storage was occurring. On the same way, the CFDCAA signals were not interpolated nor sampled in time, being acquired for the entire simulation (transient included) - all this leading to time series of 14,000 steps acquired with a regular time step of $5 \mu$ s (i.e. $70 \mathrm{~ms}$ of physical time). All this must be compared with the acquisition characteristics of the experiments, for which array signals were recorded (at the exact microphone locations) for a total of $1,966,080$ (resp. 864,256) steps acquired with a time step of $5 \mu$ s (resp. $25 \mu$ s), i.e. approximately 9.8 (resp. 21) seconds of physical time for the F2 (resp. C19) array. Given the disparity in their respective 


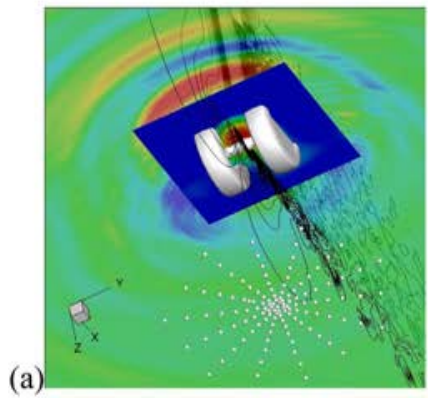

(b)

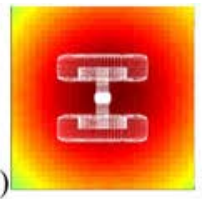

(g)

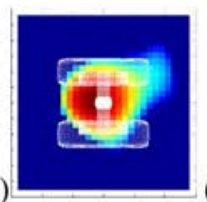

(c)

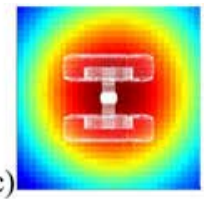

(d)
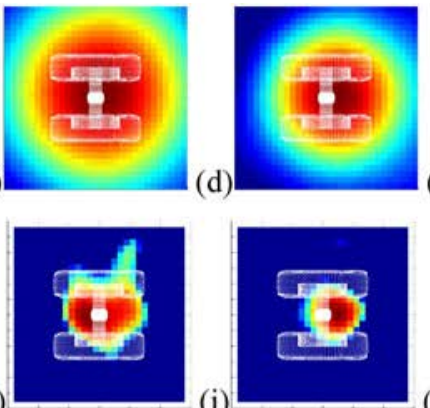

(e)
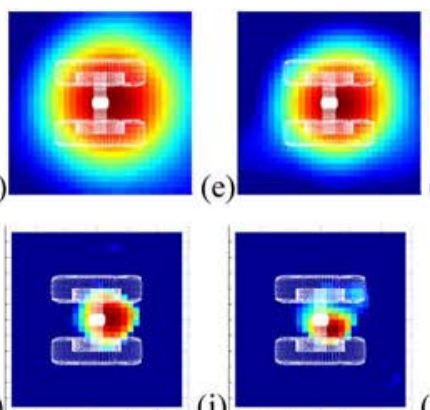

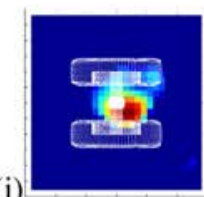

(f)

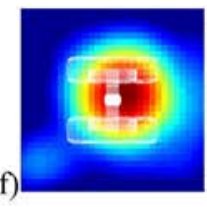

$(\mathrm{k})$

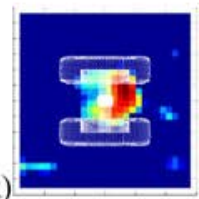

Fig. 17. Numerical characterization of the aeroacoustics by the NLG: near- to far-field noise propagation, as simulated via CFD-CAA. Further investigation of the NLG noise sources via the use of phased array localization techniques. NLG noise sources, as obtained from the numerical signals associated with the baseline (i.e. as-like F2) CFD-CAA computation (star-shape array (a), free-field, uniform flow) using either CBF ((b) to (f)) or DAMAS ((g) to (k)). Source maps obtained over a $x y$ patch of $[-0.3 \mathrm{~m} \times 0.3 \mathrm{~m}]^{2}$ via $\mathrm{CBF}$ and DAMAS exploitation of noise signal at $1 \mathrm{kHz}(\mathrm{b}$ and $\mathrm{g}$, respectively), $1.5 \mathrm{kHz}(\mathrm{c}$ and $\mathrm{h}), 2 \mathrm{kHz}(\mathrm{d}$ and i), $2.5 \mathrm{kHz}$ (e and $\mathrm{j}$ ) and $3 \mathrm{kHz}$ (f and $\mathrm{k}$ ).

(a)

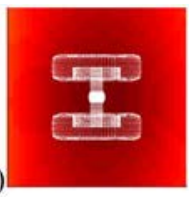

(f)

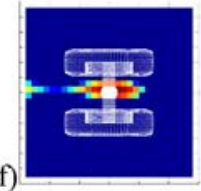

(b)

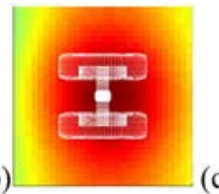

(c)

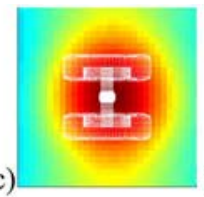

(d)

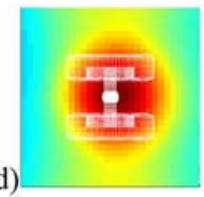

(e)

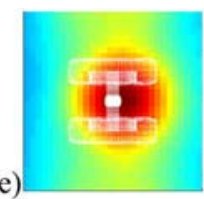

(g)

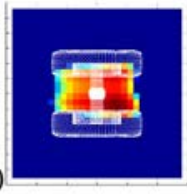

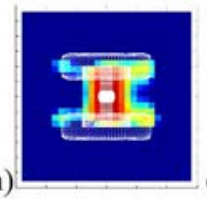

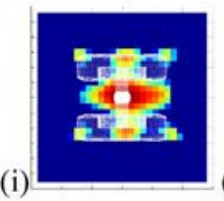

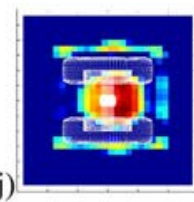

Fig. 18. Numerical characterization of the aeroacoustics by the NLG: near- to far-field noise propagation, as simulated via CFD-CAA. Further investigation ofthe NLG noise sources via the use of phased array localization techniques. NLG noise sources derived from the experimental signals associated with F2 experiment (star-shape array, closed test section, quasi-uniform confined flow), using either CBF ((a) to (e)) or DAMAS ((f) to (j)). Source maps obtained over a $x y$ patch of $[-0.3 \mathrm{~m} \times 0.3 \mathrm{~m}]^{2}$ via CBF and DAMAS exploitation of noise signal at $1 \mathrm{kHz}$ (a and f, respectively), $1.5 \mathrm{kHz}(\mathrm{b}$ and $\mathrm{g}$ ), $2 \mathrm{kHz}(\mathrm{c}$ and $\mathrm{h}$ ), $2.5 \mathrm{kHz}(\mathrm{d}$ and $\mathrm{i}$ ) and $3 \mathrm{kHz}$ (e and j).

acquisition characteristics, the numerical signals were time-averaged differently than the experimental ones, for the resulting frequency bandwidth is the same $(500 \mathrm{~Hz})$. Consequently, and because of their much shorter duration, these numerical signals were submitted to a much smaller number of averages than those applied to their experimental counterpart. Finally, both the experimental and numerical signals were FT-processed with a Hanning window applied (no overlap).

Because of its uniform mean flow, and to the confinement effects excepted, the baseline CFD-CAA simulation was similar to the experiment run in the F2 aerodynamic/closed test section wind tunnel (which incorporated a 120 microphones starshape array, located 2.6D away from the gear - see Fig. 9). On the contrary, due to its C19-based background mean flow, the alternative CFD-CAA simulation was similar to the experiment conducted in the open jet anechoic facility (which incorporated a 41 microphones cross-shape array, positioned 6.5D away from the gear). Thanks to the fact that they relied on an advanced propagation model (based on Geometrical Acoustics, GA [43,44], i.e. able to account for the realistic mean flow), both CBF and DAMAS methods could be each time adjusted to the configuration considered; whenever the latter configuration involved a uniform medium (e.g. baseline CFD-CAA computation, F2 experiment), the latter kernel reduced to the convected Helmholtz equation (homogeneous Green function). On the contrary, when the configuration involved the realistic jet (e.g. alternative CFD-CAA calculation, C19 experiment), the kernel accounted for the heterogeneous mean flow used within the CAA stage.

The CBF method was run as is, given its relative simplicity/robustness. Oppositely, and given its higher complexity/ sensitivity, the DAMAS method was tuned with care, through dedicated parametric studies, which led to a significant number of localization exercises and outputs. More precisely, the noise source focalization area was allotted various dimensions, the noise localization algorithm was run with several levels of convergence considered, and a regularization process (based on the classical approach by Tikhonov) was applied to the localization results, the regularization rate being varied.

For the sake of conciseness, the section below summarizes only a fraction of these noise source localization efforts [29], focusing in particular on the sole baseline CFD-CAA computation (i.e. as-like F2 experiment). Indeed, compared with the alternative CFD-CAA calculation (i.e. as-like C19 experiment), this particular configuration offered the advantage of being characterized by a better resolution of the array, given its higher aperture angle (i.e. array radius vs. distance to focalization 
area). Fig. 17 depicts some of the noise source maps obtained, comparing the outputs delivered by both CBF and DAMAS approaches. As one can see, whatever the localization method is, noise sources clearly emerge, without being visibly corrupted by spurious artifacts - which demonstrates the relative robustness of both methods, when it comes to exploit numerical (i.e. very short) signals. According to CBF maps, the noise sources appear as being primarily located within the wheel's area, whilst moving slightly downstream the gear when the frequency increases. Compared to CBF ones, DAMAS maps are still more explicit, revealing noise sources that are localized very accurately; there, one can appreciate how the two tonal noise sources $(1 \mathrm{kHz}, 1.5 \mathrm{kHz})$ appear to be located right between the wheels, whereas the sources of higher frequency appear to rather be positioned downstream the strut, in the wake or on the wheels themselves. These observations can be viewed in the light of what was said previously about the noise generation physics of tonal peaks, which is to be related to mechanisms occurring within the wheels' area (aeroacoustic feed-back and acoustic resonance), contrarily to those underlying the other (broadband) emissions, which are to rather be associated with the fluid-structure interactions of the turbulent flow impinging the landing gear structure.

For comparison purpose, Fig. 18 provides the experimental counterpart of Fig. 17, depicting the noise source maps derived from the F2-measured signals, once exploited through both CBF and DAMAS approaches.

As one can see, these experimental-originated noise maps are very similar to those originated from the CFD-CAA computation (see Fig. 17), although partly contaminated with spurious artifacts coming from the testing conditions. Indeed, contrarily to the calculation (whose set-up corresponded to a free-field homogeneous medium), F2 experiment was conducted within a closed test section, that is, a noisy and reverberant environment. ${ }^{15}$ Although part of these background noise and reverberation effects could be removed a posteriori by the localization procedure (esp. DAMAS), some of them contaminated the results (see the DAMAS maps at $2.5 \mathrm{kHz}$ and $3 \mathrm{kHz}$, where confinement effects clearly emerge).

Despite of these biases (whose complete removal would have needed the localization method to be enhanced with proper de-noising and de-reverberation techniques $[45,46])$, the overall agreement between the present experiment-originated noise source maps and their computational counterpart demonstrates that, although delivering noise signals of much shorter duration than experiments, unsteady CFD-based calculations can be exploited efficiently through array localization methods. This observation, which confirms the conclusions drawn by Lockard et al. in a recent study [47], speaks in favor of a more systematic use of noise localization methods within a computational context. Considering the much higher flexibility that numerical simulation offers compared to experiments (virtual arrays with unlimited number of microphones, computational set-ups de-biased from facility installation effects, etc.), this opens the door to many possibilities for numerically investigating the aircraft noise source mechanisms.

\section{Summary and concluding remarks}

The present work consisted in numerically characterizing the noise induced by an in-flight nose landing gear (NLG), through the use of advanced computational and analysis techniques; first, the NLG noise generation was reproduced by the means of an unsteady Computational Fluid Dynamics (CFD) calculation relying on the so-called Zonal Detached Eddy Simulation (ZDES) approach. The latter CFD computation was then interfaced with a Computational AeroAcoustics (CAA) calculation, so as to further simulate the NLG noise radiation up to the far-field, whilst possibly accounting for realistic flow effects (which cannot be accounted for by more traditional approaches, e.g. Integral Methods based on an Acoustic Analogy). Although some strong approximations had to be made (such as sampling and truncating the CFD-CAA interface), the resulting CFD-CAA hybrid calculation was validated against experimental records, before it was complemented by an alternative simulation (so as to numerically assess the installation effects by the experimental set-up) and post-processed using both traditional and advanced analysis techniques (so as to further investigate the underlying noise generation mechanisms).

By completing further the insights previously gained from the past experimental and numerical campaigns devoted to this particular NLG configuration (LAGooN program), the present study did not only constitute an additional step towards the numerical characterization of landing gear aeroacoustics, but it highlighted the relevance of the present methodology regarding the mitigation of aircraft noise. As an illustration of this, it is worth mentioning that the present effort was recently capitalized under the form of two benchmark test cases, which were disseminated among the community via international benchmarking initiatives ${ }^{16}$ that are sponsored by AIAA and managed by NASA, so as to improve the techniques employed worldwide for characterizing aircraft noises.

From a more global perspective, the present methodology is currently employed within several research efforts that are conducted within both European and French national frameworks, in order to numerically characterize the noise emissions by CROR (Counter Rotating Open Rotor) and HBPR (High By Pass Ratio) powered aircraft, as well as the acoustic loads induced by civil space launchers at lift-off. These follow-on works shall further highlight how advanced simulation and analysis techniques as the present ones may help in addressing realistic noise problems coming from the aerospace industry.

\footnotetext{
${ }^{15}$ Not to mention other biases, such as a haystacking effect (i.e. acoustic diffusion by turbulence) that could have been induced by the turbulent boundary layer along the walls - an effect that was however expected to be modest, given the relatively low frequency range considered here.

${ }^{16}$ Namely the Benchmark for Airframe Noise Computations and the Array Methods Workshop.
} 


\section{Acknowledgements}

Although the present work was funded by ONERA, it took direct benefit from the LAGooN project, which was supported and led by Airbus. In that regard, authors acknowledge Dr. B. Caruelle (Airbus) and Dr. E. Manoha (ONERA) for having granted them access to the LAGooN experimental database. Authors also thank Dr. L. Lopes, Dr. N. Zawodny and Dr. M. Choudhari (all from NASA/LaRC) as well as Dr. T. Imamura (JAXA) for their help in setting up a dedicated benchmark exercise within BANC workshop, on the basis of the first part of the current study (NLG noise hybrid computations). On the same way, authors acknowledge Dr. Bahr and Dr. Lockard (NASA/LaRC) for their support in proposing a dedicated benchmark exercise within the Array Analysis Methods workshop, on the basis of the second part of the present study (NLG noise sources localization).

\section{References}

[1] R. Stoker, Y. Guo, C. Streett, N. Burnside, Airframe noise source locations of a 777 aircraft in flight and comparisons with past model-scale tests, in: Proceedings of the 9th AIAA/CEAS AeroAcoustics Conference and Exhibit, AIAA paper 2003-3111, Hilton Head (USA), May 2003.

[2] E. Manoha, J. Bulté, B. Caruelle, LAGOON: an experimental database for the validation of CFD/CAA methods for landing gear noise prediction, in: Proceedings of the 14th AIAA/CEAS AeroAcoustics Conference and Exhibit, AIAA paper 2008-2816, Vancouver (Canada), May 2008.

[3] E. Manoha, J. Bulté, V. Ciobaca, B. Caruelle, LAGOON: further analysis of aerodynamic experiments and early aeroacoustics results, in: Proceedings of the 15th AIAA/CEAS AeroAcoustics Conference and Exhibit, AIAA paper 2009-3277, Miami (USA), May 2009.

[4] D.H. Neuhart, M.R. Khorrami, M.M. Choudhari, Aerodynamics of a gulfstream G550 nose landing gear model, in: Proceedings of the 15th AIAA/CEAS AeroAcoustics Conference and Exhibit, AIAA paper 2009-3152, Miami (USA), Msy 2009.

[5] T. Van de Ven, Computational and aeroacoustic analysis of A $1 \frac{1}{4}$ scale G550 nose landing gear and comparison to NASA \& UFL wind tunnel data, in: Proceedings of the 15th AIAA/CEAS AeroAcoustics Conference and Exhibit, AIAA paper 2009-3359, Miami (USA), May 2009.

[6] F.J. Souliez, L.N. Long, P.J. Morris, A. Sharma, Landing gear aerodynamic noise prediction using unstructured grids, Int. J. Aeroacoust. 1 (2) (2002) $115-135$.

[7] F. Li, M.R. Khorrami, M.R. Malik, Unsteady simulation of a landing-gear flow field, in: Proceedings of the 8th AIAA/CEAS AeroAcoustics Conference and Exhibit, AIAA paper 2002-2411, Breckenridge (USA), May 2002.

[8] D.P. Lockard, M.R. Khorrami, F. Li, Aeroacoustic analysis of a simplified landing gear, in: Proceedings of the 9th AIAA/CEAS AeroAcoustics Conference and Exhibit, AIAA paper 2003-3111, Hilton Head (USA), May 2003.

[9] L.S. Hedges, A.K. Travin, P.R. Spalart, Detached-Eddy simulations over a simplified landing gear, J. Fluid Eng. 124 (2) (2002) $413-423$.

[10] V. Vatsa, D.P. Lockard, M.R. Khorrami, Application of FUN3D solver for aeroacoustics simulation of a nose landing gear configuration, Am. Inst. Aeronaut. Astronaut. (2011) 2011-2820.

[11] S. Deck, F. Gand, V. Brunet, S. Ben Khelil, High-fidelity simulations of unsteady civil aircraft aerodynamics: stakes and perspectives. Application of zonal detached eddy simulation, Philos. Trans. Ser. A (Math. Phys. Eng. Sci.) 372 (2022) .

[12] L. Sanders, E. Manoha, S. Ben Khelil, C. François, LAGOON:CFD/CAA coupling for landing gear noise and comparison with experimental database, in: Proceedings of the 17th AIAA/CEAS AeroAcoustics Conference and Exhibit, AIAA paper 2011-2822, Portland (USA), June 2011.

[13] L. Sanders, E. Manoha, S. Ben Khelil, C. François, LAGOON: new mach landing gear noise computation and further analysis of the CAA process, in: Proceedings of the 18th AIAA/CEAS AeroAcoustics Conference and Exhibit, AIAA paper 2012-2281, Colorado Springs (USA), June 2012.

[14] W. Liu, J.W. Kim, X. Zhang, D. Angland, B. Caruelle, Landing-gear noise prediction using high-order finite difference schemes, J. Sound Vib. 332 (14) (2013) 3517-3534.

[15] J.C. Giret, A. Sengissen, S. Moreau, J.C. Jouhaud, Prediction of LAGOON landing gear noise using an unstructured LES solver, in: Proceedings of the 19th AIAA/CEAS AeroAcoustics Conference and Exhibit, AIAA paper 2013-2113, Berlin (Germany), June 2013.

[16] D. Casalino, A.F.P. Ribeiro, E. Fares, S. Nölting, Lattice-Boltzmann aeroacoustic analysis of the LAGOON landing gear configuration, AIAA J. 52 (6) (2014) $1232-1248$.

[17] F. De la Puente, L. Sanders, F. Vuillot, On LAGOON nose landing gear CFD/CAA computation over unstructured mesh using a ZDES approach, in: Proceedings of the 20th AIAA/CEAS AeroAcoustics Conference and Exhibit, AIAA Paper 2014-2763, Atlanta (USA), June 2014.

[18] A. Sengissen, J.C. Giret, C. Coreixas, J.F. Boussuge, Simulations of LAGOON landing-gear noise using lattice boltzmann solver, in: Proceedings of the 21st AIAA/CEAS AeroAcoustics Conference and Exhibit, AIAA paper 2015-2993, Dallas (USA), June 2015.

[19] E. Manoha, B. Caruelle, Summary of the LAGOON solutions from the benchmark problems for airframe noise computations-III workshop, in: Proceedings of the 21st AIAA/CEAS AeroAcoustics Conference and Exhibit, AIAA paper 2015-2846, Dallas (USA), June 2005.

[20] S. Deck, Zonal detached-eddy simulation of the flow around a high-lift configuration, AIAA J. 43 (11) (2005) 2372-2384.

[21] S. Deck, Recent improvements in the Zonal Detached Eddy Simulation (ZDES) formulation, Theor. Comput. Fluid Dyn. 26 (6) (2012) 523-550.

[22] L. Cambier, S. Heib, S. Plot, The Onera elsA CFD software: input from research and feedback from industry, Mech. Ind. 14 (3) (2013) $159-174$.

[23] L. Lopes, S. Redonnet, T. Imamura, T. Ikeda, G. Cunha, Variability in the propagation phase of CFD-based noise prediction, in: Proceedings of the 21th AIAA/CEAS AeroAcoustics Conference and Exhibit, AIAA paper 2015-2845, Dallas (USA), June 2015.

[24] S. Redonnet, G. Cunha, An advanced hybrid method for the acoustic prediction, Adv. Eng. Softw. (2015) 30-52.

[25] S. Redonnet, E. Manoha, P. Sagaut Numerical simulation of propagation of small perturbations interacting with flows and solid bodies, in: Proceedings of the 7th AIAA/CEAS AeroAcoustics Conference and Exhibit, AIAA paper 2001-2223, Maastricht (The Netherlands), May 2001.

[26] S. Redonnet, Simulation numérique de la propagation acoustique au sein d'écoulements quelconques et de structures solides par résolution des équations d'Euler [Numerical Simulation of Acoustic Propagation within Heterogeneous Flows with Solid Structures through the Solving of Euler Equations] (Ph.D. thesis), Bordeaux University, France, 2001.

[27] S. Redonnet, Y. Druon, Computational aeroacoustics of realistic co-axial engines, AIAA J. 50 (5) (2012) 1029-1046.

[28] S. Redonnet, G. Cunha, S. Ben Khelil, Numerical simulation of landing gear noise via weakly coupled CFD-CAA calculations, in: Proceedings of the 19th AIAA/CEAS AeroAcoustics Conference and Exhibit, AIAA paper 2013-2068, Berlin (Germany), June 2013.

[29] S. Redonnet, J. Bulté, Landing gear noise sources identification through an application of array methods to experimental and computational data, in: Proceedings of the 22th AIAA/CEAS AeroAcoustics Conference and Exhibit, AIAA paper 2016-2844, Lyon (France), June 2016.

[30] P. Spalart, W. Jou, M. Strelets, S. Allmaras, Comments on the feasibility of LES for wing and on a hybrid RANS/LES approach, in: Proceedings of the 1st AFSOR International Conference on DNS/LES, Ruston (USA), 1998.

[31] P. Spalart, S. Deck, M. Shur, K. Squires, M. Strelets, A. Travin, A new version of detached-eddy simulation, resistant to ambiguous grid densities, Theor Comput. Fluid Dyn. (2006) 181-195.

[32] G. Cunha, S. Redonnet, On the signal degradation induced by the interpolation and the sampling rate reduction in aeroacoustics hybrid methods, Int. J. Numer. Methods Fluids 71 (7) (2013) 910-929.

[33] S. Redonnet, D.P. Lockard, M.R. Khorrami, M.M. Choudhari, The non reflective interface: an innovative forcing technique for computational acoustics hybrid methods, Int. J. Numer. Methods Fluids 81 (1) (2016) 22-44. 
[34] G. Cunha, Optimisation d'une méthodologie de simulation numérique pour l'aéroacoustique basée sur un couplage faible des méthodes d'aérodynamique instationnaire et de propagation acoustique [Optimization of a Computational Aeroacoustic Methodology Relying on the Weak-Coupling of Unsteady Aerodynamic and Acoustic Propagation Methods] (Ph.D. thesis), Toulouse University, France, 2012.

[35] G. Cunha, S. Redonnet, Development of optimized interpolation schemes with spurious modes minimization, Int. J. Numer. Methods Fluids 80 (2) (2016) $140-158$.

[36] G. G Cunha, S. Redonnet, A novel optimization technique for explicit finite-difference schemes with application to aeroacoustics, Int. J. Numer. Methods Fluids 78 (4) (2015) 189-216.

[37] S. Redonnet, On the numerical prediction of aerodynamic noise via a hybrid approach - Part 1: CFD/CAA surfacic coupling methodology, in: Proceedings of the 16th AIAA/CEAS AeroAcoustics Conference and Exhibit, Revisited for the Prediction of Installed Airframe Noise Problems, AIAA Paper 2010-3709, Stockholm (Sweden), June 2010.

[38] R.K. Amiet, Refraction of sound by a shear layer, J. Sound Vib. 58 (4) (1975) 467-482.

[39] R.K. Amiet, Corrections of open jet wind tunnel measurements for shear layer refraction, in: Proceedings of the 2nd AIAA AeroAcoustics Conference and Exhibit, AIAA paper 75-532, Hampton (USA), 1975.

[40] S. Redonnet, J. Bulté, Numerical investigation of the refraction effects by jet flows in anechoic wind tunnels, with application to NASA/LaRC quiet flow facility, in: Proceedings of the 21th AIAA/CEAS AeroAcoustics Conference and Exhibit, AIAA paper 2015-3268, Dallas (USA), June 2015.

[41] T.F. Brooks W.M. Humphreys, A deconvolution approach for the mapping of acoustic sources (DAMAS) determined from phased microphone arrays, in: Proceedings of the 9th AIAA/CEAS AeroAcoustics Conference and Exhibit, AIAA paper 2004-2954, Manchester (UK), May 2004.

[42] T.F. Brooks, W.M. Humphreys, A deconvolution approach for the mapping of acoustic sources (DAMAS) determined from phased microphone arrays, J. Sound Vib. 294 (4-5) (2006) 856-879.

[43] S. Candel, Etudes théoriques et expérimentales de la propagation acoustique en milieu inhomogène et en movement [Theoretical and Experimental Studies of the Acoustic Propagation within a Moving and Heterogeneous Medium] (Ph.D. thesis), Paris VI University, Paris, 1977.

[44] S. Candel, Numerical solution of conservation equations arising in linear wave theory: application to aeroacoustics, J. Fluid Mech. 83 (3) (1977) 465-493.

[45] J. Bulté, Improvement of acoustic measurements with an array of microphones in aerodynamic wind tunnels, in: Proceedings of the 35th International Congress and Exposition on Noise Control Engineering (Internoise), Hawaï (USA), 2006.

[46] J. Bulté, Acoustic array measurements in aerodynamic wind tunnels: a subspace approach for noise suppression, in: Proceedings of the 12th AIAA/ CEAS AeroAcoustics Conference and Exhibit, Roma (Italy), AIAA paper 2007-3446, May 2007.

[47] D.P. Lockard, W.M. Humphreys, M.R. Khorrami, E. Fares, D. Casalino, P.A. Ravetta, Comparison of computational and experimental microphone array results for an 18\%-scale aircraft mod, in: Proceedings of the 21th AIAA/CEAS AeroAcoustics Conference and Exhibit, Dallas (USA), AIAA paper 20152990, June 2015. 